\title{
Los Sistemas aGROFORESTALES TRADICIONALES DE MÉXICO: UNA APROXIMACIÓN BIOCULTURAL
}

\author{
Ana Isabel Moreno-Calles ${ }^{1,3}$, Víctor M. Toledo ${ }^{2}$ y Alejandro Casas ${ }^{2}$ \\ 'Escuela Nacional de Estudios Superiores Unidad Morelia, \\ Universidad Nacional Autónoma de México, Morelia, Michoacán, México \\ ${ }^{2}$ Centro de Investigaciones en Ecosistemas, Universidad Nacional Autónoma de México, Morelia, Michoacán, México \\ ${ }^{3}$ Autora para la correspondencia isabel_moreno@enesmorelia.unam.mx
}

\begin{abstract}
Resumen: Se revisó información de diferentes fuentes para caracterizar la diversidad biocultural de los principales sistemas agroforestales tradicionales de México, desarrollados en distintos contextos culturales y ecológicos. Se analizaron los factores que los favorecen y aquellos que los amenazan. Se propone una clasificación general de los sistemas que considera su ubicación espacial, la intensidad de manejo del sistema y el contexto ecológico y biocultural en el que se desarrollan. Para ilustrar los distintos tipos de sistemas se revisan el tlacolol de Guerrero, la kool de la Península de Yucatán, el metepantle de Tlaxcala, las chinampas del Valle de México, el calal del suroeste de Tlaxcala, el sistema milpa-cactáceas columnares del Valle de Tehuacán, el huamil en Guanajuato, los oasis de la Península de Baja California, el kuojtakiloyan en la Sierra Norte de Puebla, el te 'lom en San Luis Potosí, los cacaotales de la Chontalpa en Tabasco y del Soconusco en Chiapas, el ekuaro de Michoacán, los patios de Oaxaca y los solares de Puebla y Yucatán. Se discuten los beneficios ecológicos y económicos de estos sistemas y se proponen estrategias que permitan apoyar los procesos locales y regionales en defensa de la diversidad biocultural.
\end{abstract}

Palabras clave: etnoecología, manejo de plantas, Mesoamérica, sistemas agrícolas tradicionales.

\begin{abstract}
We reviewed information about traditional agroforestry systems of Mexico and its biocultural diversity. The review included the slash and burn agricultural systems: kool in the Yucatán Peninsula and tlacolol in Guerrero; the terrace called metepantli in Tlaxcala; the drained fields chinampa in the Valley of México and calal in southwestern Tlaxcala; the arid land systems milpa-chichipera in the Tehuacan Valley, huamil in Guanajuato and oasis (oases) in the Baja California Peninsula; the agroforests kuojtakiloyan in the Northern Sierra of Puebla, te 'lom in San Luis Potosí and cacaotales in Soconusco region in Chiapas and Chontalpa region in Tabasco; and the homegardens ekuaro in Michoacán and other homegardens in Puebla, Oaxaca and Yucatán. The discussion emphasizes the importance of traditional agroforestry systems and strategies for their maintenance.
\end{abstract}

Key words: ethnoecology, Mesoamerica, plant management, traditional agricultural systems.

$\mathbf{T}$ iempo, naturaleza y cultura son elementos esenciales de una fructífera relación que ha dado como resultado una propiedad emergente de los sistemas ecológicos, la diversidad biocultural (DBC). La expresión más conspicua de ésta la constituyen el conjunto de variedades, especies, espacios, sistemas y paisajes intencionalmente creados, mantenidos y utilizados por grupos culturales, comunidades, familias e individuos durante largos períodos de tiempo; pero también son parte de esta DBC, las lenguas, las creencias, los rituales, las tradiciones, las reglas de uso, los conocimientos y las innovaciones desarrolladas en el tiempo (Toledo y Barrera-Bassols, 2008; Pretty et al., 2009).

Nuestro país tiene una gran riqueza de pueblos origi- narios, que han interactuado por miles de años con la alta diversidad biológica de este territorio; derivado de ello, existen expresiones notables de la DBC, tales como: (1) la modificación y creación de los paisajes a partir de la selección, quema, siembra, transplante y protección de plantas útiles en casi todos los tipos de vegetación (Denevan, 1992; Gómez-Pompa, 1991; Casas et al., 1999, 2007); (2) las cerca de 5,500 especies descritas con algún tipo de uso por diversas culturas (Caballero y Cortés, 2012); y (3) la domesticación de más de 200 especies (Casas et al., 2007), así como las formas de manejo incipiente de al menos 700 especies de plantas nativas de importancia cultural regional o local (Casas et al., 2007; Blancas et al., 2010). 
Uno de los principales escenarios donde se ha generado y desarrollado la DBC referida, son los sistemas agroforestales (SAF), en particular los sistemas tradicionales o de la "tradición moderna", como los definen Toledo y BarreraBassols (2008). Los sistemas agroforestales tradicionales y sus prácticas son formas de uso del suelo con una larga historia de manejo en México, incluso precolombina (Krishnamurthy y Ávila, 1999). En estos sistemas se desarrolla: (1) la preservación selectiva de componentes forestales o silvícolas (silvestres), principalmente leñosos y perennes, los cuales pueden estar bajo manejo incipiente mediante prácticas de tolerancia, fomento, protección y siembra de grupos de plantas y especies particulares (Casas et al., 1997, 2007); (2) el manejo de elementos agrícolas que incluyen plantas perennes o anuales, cultivadas y con niveles avanzados de domesticación; (3) en algunas ocasiones, el manejo de animales silvestres, en proceso de domesticación o domesticados y, (4) unidades sociales de producción que realizan el manejo de los distintos componentes para maximizar las interacciones ecológicas y ecológicas entre los elementos forestales y agrícolas del sistema dentro de un contexto ecológico, cultural y económico particular (Nair, 1997). Para la agroforestería, el enfoque de estudio se da en los componentes del sistema (forestales, agrícolas y unidades sociales); pero ante todo, en las emergencias resultantes de la interacción entre estos componentes (Krishnamurthy y Ávila, 1999). En la presente revisión examinamos algunos de los sistemas agrícolas tradicionales de México desde el enfoque de la agroforestería, se identifican varios sistemas agrícolas tradicionales que no habían sido considerados previamente como agroforestales, poniendo énfasis en el manejo de los elementos forestales de estos sistemas, así como en las interacciones agroforestales y de la importancia para el sistema en su conjunto.

El importante papel de los SAF tradicionales en el mantenimiento y desarrollo de la DBC se debe a que: (1) se encuentran integrados a estrategias múltiples de uso y manejo de la diversidad y proveen diversos beneficios a los seres humanos a escala local, regional y global (Alcorn, 1990; Toledo, 1990; Schroth et al., 2004); (2) conservan especies nativas, endémicas y de importancia cultural (Alcorn, 1990; Schroth et al., 2004; Bhagwat et al., 2008); (3) integran y recrean las cosmovisiones, los conocimientos, las prácticas y las reglas de uso (Toledo, 2002; Simons y Leakey, 2004) y, (4) son escenarios para la innovación de las estrategias de manejo y de domesticación de especies y paisajes y, por lo tanto, áreas de conservación y continuo desarrollo de DBC (Casas et al., 2007; Blancas et al., 2010). No obstante, los SAF de los pueblos de México están bajo la presión de diversos factores socio-ecológicos que han determinado cambios negativos en la DBC. Entre éstos, son de destacarse la disminución y cambios en la riqueza, composición y diversidad de los sistemas a escala local y de paisaje, así como la pérdida y transformación de cosmovisiones, conocimientos y prácticas tradicionales que sostienen a tal diversidad, o incluso la pérdida de los sistemas.

Afortunadamente, en la última décadas se han incrementado los estudios de estas formas de uso de la tierra y actualmente se están desarrollando estrategias conjuntas entre organizaciones no gubernamentales, organizaciones campesinas, universidades y centros de investigación para la caracterización, inventario, revaloración y mejoramiento de los SAF, los cuales empiezan a ser reconocidos como parte de los patrimonios de los pueblos de México (Boege, 2008). Estos antecedentes son la base necesaria para la atención de problemas ambientales globales, como son la pérdida y transformaciones indeseables de la diversidad biológica y cultural, la erosión genética, la crisis alimentaria y el cambio global; pero también para contribuir a la supervivencia, permanencia, desarrollo y defensa de la identidad y de los territorios de los pueblos de nuestro país. En este contexto, el presente estudio analiza: (1) ¿Cuáles son los SAF tradicionales de México?, ¿cómo son, qué pueblos los manejan, qué diversidad biocultural mantienen y dónde están?; (2) ¿ cuáles son los beneficios ecológicos, sociales y económicos de estos sistemas y su diversidad?; (3) ¿ cuáles son las transformaciones y amenazas que enfrentan estos sistemas y la diversidad biocultural que alojan? y, (4) ¿qué estrategias se pueden desarrollar para apoyar los esfuerzos de las comunidades campesinas y organizaciones para su mantenimiento?

Los objetivos de esta investigación fueron: (1) caracterizar a los principales SAF tradicionales de los pueblos de México y la diversidad biocultural que mantienen; (2) analizar las amenazas a la que se enfrentan y, (3) proponer estrategias que permitan apoyar los procesos locales y regionales de defensa de la diversidad biocultural.

\section{Métodos}

Elección y clasificación de los sistemas agroforestales tradicionales. Para identificar los SAF tradicionales más relevantes del país se hizo una revisión y sistematización de la literatura acerca de los sistemas agrícolas tradicionales. Son particularmente importantes los trabajos de Hernández-Xolocotzi (1977), Wilken (1987), Rojas Rabiela y Sanders (1985), Rojas Rabiela (1991) y Whitmore y Turner II (2001).También se revisaron los trabajos previos acerca de sistemas agroforestales tradicionales en México que se enfatizan en plantas, tales como los trabajos de Alcorn (1990), Puig (1994) y Krishnamurthy y Ávila (1999). Los SAF cuya descripción se seleccionó tomó en cuenta: (1) que fueran representativos de los grandes tipos de sistemas identificados; (2) que fueran sistemas agrícolas con largo tiempo de permanencia en México incorporando prácticas agroforestales para el manejo de árboles, arbustos y hierbas del bosque original y, (3) que fueran sistemas utilizados en la actualidad. Se hicieron búsquedas especializadas por el nombre genéri- 
co para los diferentes SAF elegidos, estos fueron: (1) sistemas agroforestales de descanso largo (roza, tumba y quema, tlacolol, kool, agricultura itinerante, slash and burn agriculture, shifting agriculture, swidden agriculture); (2) terrazas (metepantli, terrace, terracing, sloping field, cross-channel field); (3) sistemas de humedales (agricultura de campos elevados, calal, chinampa, campos drenados, drained field, raised field); (4) sistemas de zonas áridas (sistemas agroforestal milpa-chichipera, huamil y oasis); (5) agrobosques (agroforest, kuojtakiloyan, te lom y cacaotal) y, (6) huertos (calmil, solar, ekuaro, homegarden). Con base en la revisión se identificaron los siguientes criterios que fueran útiles para clasificar los sistemas agroforestales descritos en el texto:

(i) Ubicación del sistema. En México, la estrategia más común de manejo del paisaje incluye tres componentes principales: el monte, la parcela y el huerto o solar, y es posible identificar sistemas agroforestales en estas tres unidades. Esta clasificación es dinámica y frente a cambios biofísicos, culturales, sociales y económicos, la parcela puede pasar a ser monte y el monte puede eventualmente ser parcela o la parcela solar.

(ii) Intensidad de manejo. Este criterio considera la duración del descanso de la tierra o barbecho, el manejo del agua, suelo y biota del sistema, el esfuerzo humano y animal invertido, y el tipo de instrumentos e insumos utilizados en el sistema. Estos elementos tienen influencia en la composición de las especies perennes y anuales, agrícolas y forestales de los SAF. Con base en ellos se describieron tres categorías: (a) sistemas de baja intensidad, aquellos en los que el descanso de la tierra excede al período de cultivo, son de temporal, el suelo es manejado mediante herramientas manuales; (b) sistemas de mediana intensidad, con descansos cortos, el período de cultivo excede al de descanso y por lo tanto, la fase forestal del sistema es compuesta principalmente por hierbas, arbustos y árboles de rápido crecimiento; son de temporal o con riego de auxilio, con suplementaciones de nutrientes al suelo y empleo de la yunta en las labores agrícolas y forestales; (c) sistemas intensivos, en los que no hay descanso de la tierra, se emplea el auxilio de animales para las labores agrícolas y en donde es posible el uso de maquinaria.

(iii) El contexto ecológico. Las diversas condiciones climáticas, morfo-edafológicas y de vegetación en México han planteado condiciones propicias y adversas para el desarrollo y permanencia de los sistemas agroforestales. Se describen sistemas en cuatro condiciones principales: (a) zonas templadas, (b) zonas áridas y semiáridas, (c) zonas cálidohúmedas y (d) zonas de humedales.

(iv) El contexto biocultural. La cultura guía las interacciones entre los seres humanos y su medio e influye en el manejo de los sistemas agroforestales. Incluye una cosmovisión y creencias particulares, conocimientos, prácticas de manejo, preferencias por el manejo de especies particulares y grupos de ellas que determinan la composición de los SAF. Se presentan ejemplos para algunos grupos culturales: nahua, me' phaa, ñu savi, purhépecha, teenek, ñañu, zapoteco, maya, mestizos y rancheros.

Caracterización de los sistemas y su diversidad biocultural. Se profundizó en la descripción de SAF tradicionales, su ubicación espacial y temporal, el contexto ecológico y cultural, las reglas de uso, los tipos de sistemas similares, las prácticas agroforestales, los usos y formas de manejo de las especies que los componen, todo ello para caracterizar la diversidad biocultural. Se adoptó el sistema de nomenclatura de The International Plant Name Index (2012) para identificar los nombres de las especies descritas. Se consultó la obra de Martínez (1979) para una aproximación a la identificación de especies referidas en algunas fuentes sólo con nombres comunes. Se incorporó información sobre las comunidades donde se encuentran los SAF descritos, así como sobre las actividades que realizan centros académicos y organizaciones no gubernamentales que colaboran con éstas.

\section{Resultados}

Se identificaron 20 diferentes nombres de sistemas agroforestales actualmente en uso en el país (tlacolol, kool, milpa, terraza, metepantle, milpa-chichipera, huamil, oasis, tajos, milpa-mezquital, palma-milpa, calal, chinampa, télom, kuojtakiloyan, cacaotal, huerto, solar, patio, ekuaro). En la presente revisión se eligieron algunos de tales sistemas para construir un esquema general de clasificación y para ejemplificar las categorías generales de tal esquema de clasificación (Figura 1, Cuadro 1): (1) Sistemas agroforestales de parcela de baja intensidad en zonas templadas, subhúmedas y cálido-húmedas, practicados por comunidades nahuas, me phaa, ñu savi, mayas y mestizas. En este tipo se incluyen a los sistemas agroforestales de tlacolol de Guerrero y kool de la Península de Yucatán. (2) Sistemas agroforestales de parcela y de casa de mediana intensidad, y de alta intensidad desarrollados en zonas áridas y semiáridas, manejados por grupos mestizos y rancheros. Se describen el sistema agroforestal de milpa-chichipera en el Valle de Tehuacán, el huamil en Guanajuato y los oasis de Baja California Sur. (3) Sistemas agroforestales de monte, de media a alta intensidad, ubicados en condiciones cálido-húmedas y semicálido-húmedas, que son manejados por grupos mestizos, mayas, teenek y nahuas. Se incluyen en esta categoría a los llamados agrobosques, tales como el cacaotal, el te 'lom y el kuojtakiloyan. (4) Sistemas agroforestales de parcela muy intensivos, desarrollados en las laderas de zonas templadas y áridas, son manejados por grupos nahuas y mestizos. El ejemplo que se describe es el metepantle de Tlaxcala. (5) Sistemas agroforestales de parcela y de casa de alta intensidad, que se desarrollan en los humedales del centro de México, , y que actualmente son practicados por grupos mestizos. En estos sistemas se incluye a la chinam$p a$ del Centro de México y al calal del suroeste de Tlaxca- 


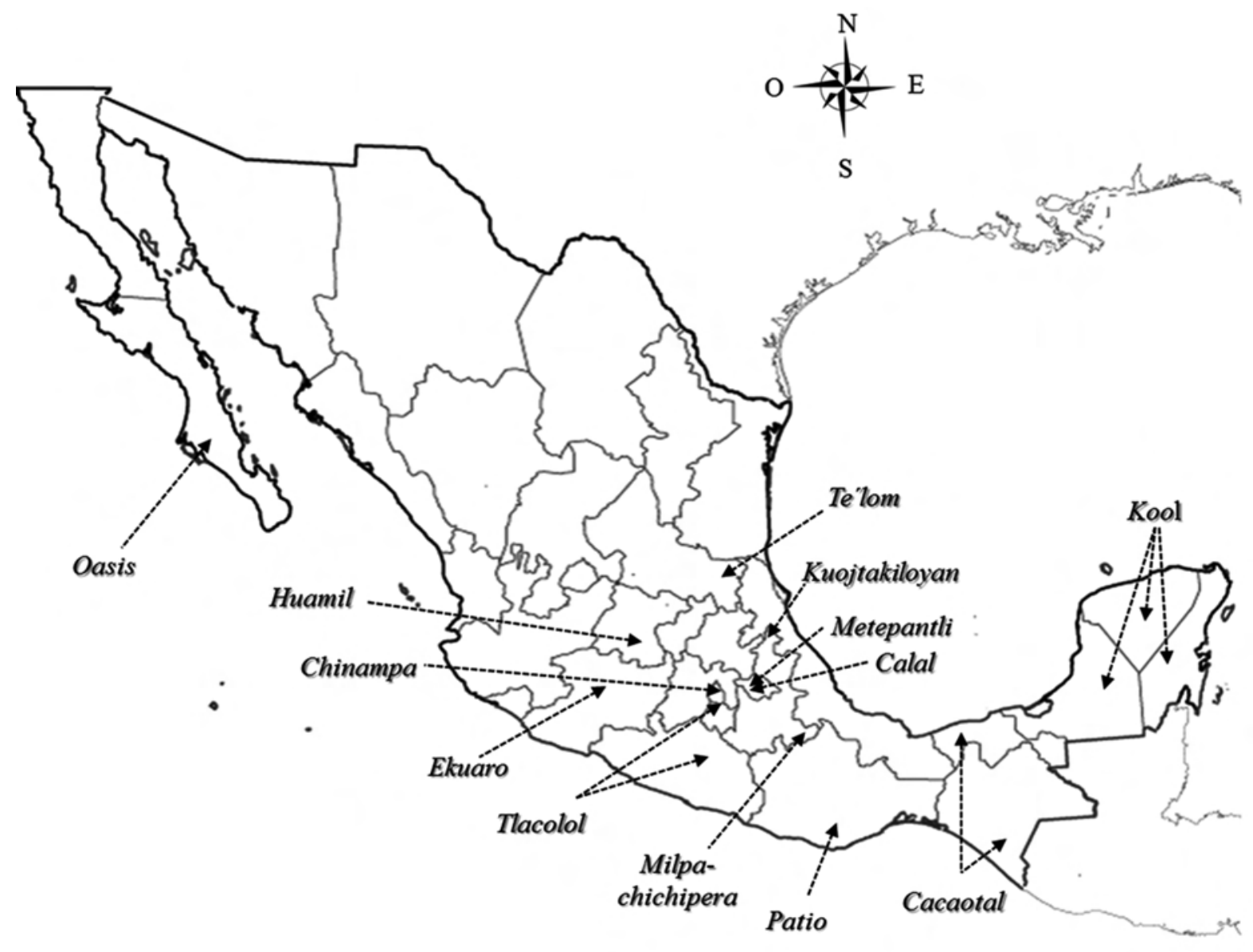

Figura 1. Ubicación de los Sistemas Agroforestales Tradicionales de México revisados.

Cuadro 1. Clasificación de los sistemas agroforestales tradicionales revisados en este trabajo.

\begin{tabular}{|c|c|c|c|c|}
\hline UBICACIÓN & INTENSIDAD DE MANEJO & AMBIENTE & GRUPO HUMANO & SISTEMA \\
\hline \multirow[t]{8}{*}{ Parcela } & baja intensidad & sub-húmedo & $\begin{array}{c}\text { nahuas, mepha'a, } \\
\tilde{n} u \text { savi, mestizos }\end{array}$ & tlacolol \\
\hline & & cálido-húmedo & mayas & kool \\
\hline & media intensidad & semiárido & mestizos & milpa-chichipera \\
\hline & & & mestizos & huamil \\
\hline & & & ñañú & terrazas \\
\hline & alta intensidad & templado & nahuas, mestizos & metepantle \\
\hline & & humedal & mestizos & chinampa \\
\hline & & & mestizos & calal \\
\hline \multirow[t]{3}{*}{ Monte } & media intensidad & cálido-húmedo & teenek & te $l o m$ \\
\hline & alta intensidad & semicálido- húmedo & nahuas & kuojtakiloyan \\
\hline & & cálido-húmedo & mayas & cacaotal \\
\hline \multirow[t]{4}{*}{ Casa } & Alta intensidad & templado & purhépecha & ekuaro \\
\hline & & árido & rancheros & oasis \\
\hline & & semiárido & mestizos & huerto \\
\hline & & cálido-húmedo & $\begin{array}{c}\text { mayas } \\
\text { zapotecos }\end{array}$ & $\begin{array}{l}\text { huerto } \\
\text { patio }\end{array}$ \\
\hline
\end{tabular}


la. (6) Sistemas agroforestales de casa intensivos, que se desarrollan en condiciones templadas, áridas, semiáridas, subhúmedas y cálido húmedas y por casi todos los grupos humanos que se revisaron, se presentan ejemplos de grupos purhépecha, zapotecos, mestizos y mayas. Los nombres locales de los ejemplos presentados en el texto son solar, huerto, ekuaro y patio.

El tlacolol de Guerrero y kool o milpa en la Península de Yucatán. Una de las formas de colonización agrícola de las zonas boscosas de Mesoamérica lo han sido los sistemas agrícolas que incluyen el aclareo de vegetación, seguido generalmente del empleo del fuego, el cultivo por periodos cortos y su alternancia con un descanso forestal que excede al periodo agrícola (Thrupp et al., 1997). A partir de estas técnicas se han desarrollado sistemas agroforestales conocidos en la literatura por: (1) el mayor tiempo de descanso en relación al período de cultivo de los terrenos y (2) la alternancia entre estos periodos. Se han denominado sistemas de descanso largo, agricultura itinerante y shifting cultivation. Estos sistemas son conocidos frecuentemente por el método de aclareo y limpieza del terreno, se han nombrado como sistemas de roza, tumba y quema; desmonte y quema; slash and burn agriculture y swidden agriculture (Rojas Rabiela, 1991; Thrupp et al., 1997; Whitmore y Turner II, 2001). Estas formas de agricultura podrían tener una antigüedad de 4,500 años (Rojas Rabiela, 1991). Actualmente este tipo de sistemas se distribuye en terrenos montañosos de laderas muy pronunciadas, principalmente en el bosque tropical caducifolio de los estados de Morelos, Guerrero, Oaxaca y Michoacán; así como en las áreas planas con pendientes suaves del bosque tropical con suelos delgados y pobres; y en las regiones con suelos calcáreos, como es la Península de Yucatán (Rojas Rabiela, 1991; Toledo et al., 2003). Estos son sistemas agroforestales debido a que: (a) cuando se roza la vegetación, suelen dejarse en pie árboles y arbustos silvestres o sus tocones que pueden rebrotar, lo que facilita la recuperación del bosque y el aprovechamiento de las especies que se dejan en pie (Rojas Rabiela, 1991). Esta puede haber sido una de las primeras y más importantes prácticas agroforestales en Mesoamérica (Whitmore y Turner II, 2001) y (b) alternan uno o dos ciclos agrícolas con un descanso largo para permitir la recuperación del bosque y la fertilidad del suelo, y durante los períodos de barbecho algunas especies son promovidas en la sucesión de la vegetación (Whitmore y Turner II, 2001). Una variante de este sistema es el tlacolol, que generalmente se cultiva por un ciclo agrícola al año durante el temporal, principalmente para la siembra de diversas variedades de maíz, frijol y calabaza; se alternan períodos cortos de cultivo con periodos largos de barbecho que permitan la recuperación de la vegetación y de la fertilidad del suelo. El tlacolol se ha asociado a climas cálido subhúmedo y templado, dominados principalmente por bosque tropical caducifolio, y en menor cantidad por bosques de encinos y de pino-encino. Existen registros de tlacololes en las laderas de la Sierra Madre Oriental, Sierra Madre del Sur, la Sierra Norte de Chiapas y en la Sierra Volcánica Transversal (Rojas Rabiela, 1991; Casas et al., 1994; Lambert, 1996; Barrera-Cristobal, 1999; Whitmore y Turner II, 2001; Áviles et al., 2012). En las regiones conocidas como Centro-Montaña y Montaña de Guerrero es practicado hasta la actualidad por comunidades nahuas, méphaa (tlapanecos) y ñu savi (mixtecos) (Casas et al., 1994; Áviles et al., 2012). En estos sistemas se han registrado prácticas agroforestales y de uso y manejo de plantas de importancia biocultural. Los tlacololeros acostumbran a dejar en pie selectivamente algunos tocones y árboles porque facilitan la recuperación de la vegetación, o bien porque protegen o favorecen la propagación de otras plantas que son valoradas por sus usos y funciones; esto deriva generalmente en la práctica agroforestal conocida como árboles aislados o árboles intersticiales en campos de cultivo. En Morelos (Tepoztlán) y Guerrero (Acatlán) se han descrito especies de árboles y arbustos manejadas de esta manera tales como copal (Bursera bipinnata, B. glabrifolia, B.copallifera), pochote (Ceiba aesculifolia), lináloe (Bursera linanoe), mezquite (Prosopis laevigata), huizache (Acacia farnesiana), cacaloxochitl (Plumeria rubra) (Rojas Rabiela 1991). Los agricultores suelen crear cercas vivas o linderos de vegetación alrededor de sus parcelas, práctica agroforestal aparentemente adoptada en este sistema a partir de la Conquista, para evitar el ingreso del ganado a los campos de cultivo durante el ciclo agrícola (Rojas Rabiela, 1991). En el trabajo de Casas et al. (1994), realizado en comunidades $\tilde{n} u$ savi de Alcozauca, los autores identificaron que el yuntú $n$ dashi (término utilizado para el tlacolol en mixteco) se realizaba desde los 1,400 hasta 2,900 m s.n.m., en el bosque tropical caducifolio y en el bosque de encino y de pino-encino. Los campesinos alternan los periodos de descanso de acuerdo al tipo de vegetación donde se desarrolla este SAF, describiendo un periodo de recuperación de entre 12 a 20 años; la presencia de los tocones y árboles en pie dejados durante la roza favorece el proceso de recuperación de la vegetación. En particular, varias especies como: ocote blanco (Pinus ayacahuite), ocote rojo (Pinus montezumae), encino blanco (Quercus magnoliifolia), tepeguaje (Lysiloma acapulcense), sabinos (Juniperus flaccida y J. deppeana), guaje (Leucaena esculenta), agave (Agave cupreata), palma (Brahea dulcis), son árboles o arbustos dejados deliberadamente en pie en estos sistemas debido a que son apreciados por sus hojas y frutos para consumo del ganado, además de su leña y madera. El tlacolol es también practicado hasta la actualidad en comunidades mestizas; en un muestreo reciente realizado en la región Centro-Montaña de Guerrero, en el ejido del Jagüey (Figura 2), los autores encontraron en el SAF de tlacolol 17 especies nativas manejadas como árboles aislados, todas con usos y funciones claramente identificadas por los campesinos, entre estas especies destacan: (1) el guaje rojo (Leucaena esculenta) muy apreciada por sus vainas y 


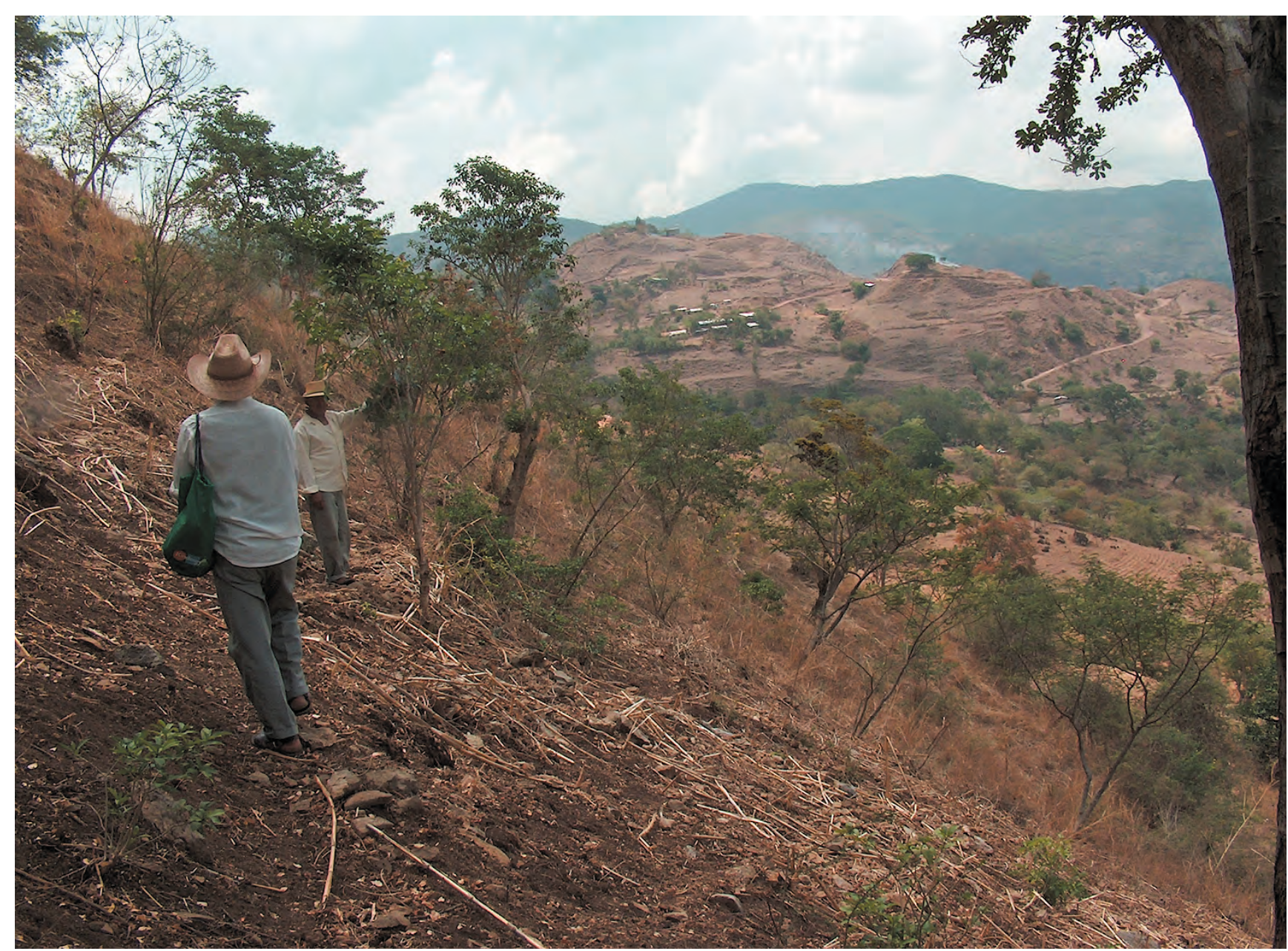

Figura 2. El Tlacolol en el ejido el Jagüey, Guerrero.

semillas comestibles, comercializadas e intercambiadas en el mercado regional de Chilapa y que además proporcionan abono para los campos de cultivo. Este guaje suele ser sembrado en los terrenos de cultivo e incluso propagado en el vivero comunitario. (2) Amate amarillo (Ficus petiolaris), un árbol escaso en la comunidad que es sembrado por los campesinos, pero éstos también consideran que los murciélagos y los tlacuaches lo propagan en los campos. (3) El copalcahuite (Bursera copallifera), empleado para manufacturar monturas y yugos, además de ser apreciados por su resina. Estas especies suelen ser protegidas del ganado y en algunos casos propagadas por medio de semillas (GarcíaMartínez, 2012). Lambert (1996) describió este sistema en una comunidad de migrantes ubicada en la porción oeste de Guerrero, en el Ejido de La Laja. Cuando se realiza el aclareo de la vegetación en este sitio se protegen la parota (Enterolobium cyclocarpum), guayaba (Psidium guajava), ciruelo (Spondias purpurea), guaje (Leucaena esculenta) y pinzán o guamúchil (Pithecellobium dulce), y otros que son promovidos mediante su cultivo, como la anona (Annona reticulata Sieber), zapote negro (Diospyros digyna) y tama- rindo (Tamarindus indica). Estos árboles proporcionan frutos y otros materiales que son colectados durante el período de descanso de las parcelas, actividad que hasta el momento en que se realizó el estudio, era regulada por las autoridades locales para evitar intervenir con el proceso de recuperación de la vegetación y de la fertilidad del suelo.

Otro ejemplo de este sistema se encuentra en la Península de Yucatán, donde: (a) el paisaje predominante es una llanura de roca caliza con superficie pedregosa y delgada capa de suelo; (b) la mayor parte de los nutrientes se encuentra disponible en la vegetación y no en el suelo; (c) domina el clima cálido-subhúmedo con una fuerte presencia de huracanes a la mitad del año; (d) es seco en el noroeste y la humedad se incrementa hacia el sureste; (e) la costa oriental y el interior son dominados por selva mediana subperennifolia y hacia el sur por selva alta; (f) no existen fuentes superficiales de agua disponibles y son aprovechadas las fuentes subterráneas como los cenotes; (g) es una región con alta diversidad indígena y los mayas fueron aparentemente los primeros colonizadores de estos sitios (Dahlin, 1985; Flores y Espejel, 1994). 
En esta región, la milpa tiene varias etapas reconocidas y manejadas por los campesinos mayas. La selva madura ( $\mathrm{su}$ huy k'aax) se desmonta para la siembra mediante la roza de arbustos, matorrales, bejucos, malezas y ramas bajas de los árboles, seguida por la tala de árboles y arbustos mayores, se dejan los tocones y protegen a algunas especies útiles por medio de podas y guardarrayas para el fuego; las principales especies protegidas son: nanche ó chi' (Byrsonima sp.), cocoyol (Acrocomia mexicana), grosella (Phyllanthus acidus), kopo' (Ficus cotinifolia), ox (Brosimum alicastrum), palma de guano (Sabal yapa) y chicozapote (Manilkara zapota) (Emerson, 1953; Hernández-Xolocotzi y Padilla, 1980; Rojas Rabiela, 1991; Terán y Rasmussen, 1994; Arias, 1995; Remmers y Ucan, 1996). Además, se construyen cercos vivos conocidos como k'alche' y se realiza la tolerancia de grandes franjas de vegetación de bosque tropical de entre 10 y 20 metros de amplitud que bordean las parcelas cultivadas, estos bordes son conocidos con el nombre de t'olche' y tienen un estatus de protección dentro de los reglamentos comunitarios (Remmers y De Koeijer, 1992; Remmers y Ucan, 1996). Las especies arbóreas que suelen ser más abundantes en esta práctica agroforestal son el kitance' (Caesalpinia gaumeri), ya'ax eek' (Pithecellobium leucospermum) y saak boo (Coccoloba cozumelensis) (Remmers y De Koeijer, 1992). El t'olche' tiene importantes funciones, como la delimitación de los terrenos, el aprovisionamiento de leña, materiales para construcción, artículos rituales y medicinales, barrera rompevientos, como atractor de lluvia, además de favorecer la recuperación de la vegetación cuando las parcelas se dejan en descanso (Remmers y De Koeijer, 1992). La etapa conocida como kool describe un concepto similar a la milpa entre las culturas nahuas; en ésta se llevan a cabo tres ciclos agrícolas antes de dejarse en descanso. En las milpas mayas existe una gran riqueza y heterogeneidad espacial y temporal de cultivos y de sus variedades que incluyen como cultivo principal al maíz, asociado a frijol, calabaza, camote, yuca, jícama, macal, jitomate, hortalizas y plantas introducidas como melón, sandía y pepino (Rojas Rabiela,1991; Toledo et al., 2008). El trabajo de Terán y Rasmussen (1994) documenta la presencia de 25 especies de cultivos y 98 variedades de éstas en las milpas de Xocen, Yucatán. La última etapa es denominada hubche', que es el barbecho o descanso largo, constituida por seis fases reconocidas con nombres mayas que permiten la recuperación de la vegetación y de la fertilidad del suelo y que generan un paisaje diverso con parcelas en distintas fases sucesionales (Barrera-Bassols y Toledo, 2005).

Por largo tiempo predominó la idea de que las etapas de barbecho no proporcionaban recursos y tampoco involucraban prácticas de manejo; sin embargo, diversos estudios han documentado que las selvas secundarias son capaces de proporcionar un gran número de recursos en sus distintas etapas. Por ejemplo, el trabajo de Toledo et al. (1995) mostró que las selvas secundarias manejadas bajo el sistema de barbecho largo son espacios que alojan la mayor cantidad de especies de plantas útiles, principalmente hierbas y arbustos medicinales, en comparación con las selvas maduras y las milpas. Bernice (2010) documentó que las selvas secundarias tempranas son espacios atractivos para especies animales valoradas por los mayas como el pavo de monte, el venado y el pecarí. La complejidad de la estrategia maya de manejo múltiple y de las técnicas silvícolas no está sólo presente en los sistemas agroforestales de milpa; es más bien complemento de otros sistemas y técnicas presentes en su territorio. El estudio de Gómez-Pompa (1987) por ejemplo, documenta prácticas silvícolas relacionadas como la introducción de árboles útiles a los cenotes, la germinación de semillas, la siembra de árboles, la selección de árboles de especies silvestres en los huertos mayas, la conservación de parches de vegetación y la selección e introducción de especies útiles en los bosques o selvas (Rojas Rabiela, 1991; Remmers y De Koeijer, 1992; Remmers y Ucan, 1996; Toledo et al., 2003, 2008; Barrera-Bassols y Toledo, 2005).

Actualmente, tanto el sistema de tlacolol en Guerrero como la milpa maya en la Península de Yucatán se enfrentan a importantes problemáticas: (1) el acortamiento de los períodos de descanso; (2) la pérdida paulatina de prácticas agroforestales como los cercos vivos, árboles aislados y bordes de vegetación; (3) la disminución de las prácticas de manejo de las especies toleradas, fomentadas o protegidas; (4) disminución de la cobertura vegetal; (5) el uso de agroquímicos para suplementar a los cultivos; y (6) la pérdida de variedades de cultivos específicos para el sistema.

Los sistemas milpa-cactáceas columnares en el Valle de Tehuacán, el huamil en Guanajuato y los oasis de la Península de Baja California. Cerca del 60\% del territorio de México son zonas áridas, semiáridas y subhúmedas (Rzedowski, 1993), en donde viven grupos originarios del país (Casas et al., 2010). En las zonas secas de México se han descrito sistemas agroforestales manejados por los nañú en el Valle del Mezquital en Hidalgo y por las comunidades pápago en el desierto de Sonora (Altieri y Toledo, 2005), los tajos de la Sierra de Xichú en Guanajuato (Hoogesteger-van Djik, 2012) y los mezquitales-milpa de las zonas aluviales del Valle de Tehuacán (Vallejo-Ramos com. pers.). Los autores de este trabajo actualmente estudian sistemas de palma (Yucca sp.) en combinación con milpa en el Carmen Tequexquitla, Tlaxcala.

Los bosques de cactáceas columnares de las zonas áridas del Valle de Tehuacán son clareados para practicar agricultura por comunidades nahuas, popolocas, ixcatecas, mazatecas, mixtecas, cuicatecas y mestizas, principalmente. En ellos se ha incorporado el policultivo de milpa, que incluye variedades criollas de maíz, fríjol y calabaza. Es una práctica común la tolerancia de especies útiles de cactáceas columnares, agaváceas y otras especies nativas en los contornos de las parcelas, en pequeñas franjas, islas de vegetación 
o incluso como individuos aislados en el centro de las parcelas (Casas et al., 2008; Moreno-Calles et al., 2010). En ocasiones se realiza la siembra de propágulos vegetativos o el transplante de plántulas e individuos juveniles dentro de los campos de cultivo (Moreno-Calles et al., 2010). En un estudio realizado en la comunidad de San Luis Atolotitlán, Puebla, los autores encontraron que en el SAF de milpachichipera (Figura 3), nombrado así por la dominancia del chichipe (Polaskia chichipe), existían 122 especies de plantas, de las cuales el $90 \%$ son nativas y el $75 \%$ de todas ellas son útiles, principalmente con uso forrajero, combustible y alimenticio, el $52 \%$ son manejadas a partir de prácticas de manejo in situ y ex situ, y el $26 \%$ tienen valor comercial o de intercambio. Las especies con mayor dominancia y valor local en las parcelas agrícolas son: panoxochitl (Montanoa sp.) por ser forraje de buena calidad; nopalli (Opuntia pilifera) por sus cladodios tiernos comestibles (nopalitos), maguey de potrero (Agave scaposa) porque evita la erosión y es hábitat de especies de gusanos comestibles muy valoradas, conocidas localmente como condachos y chichi- pe (Polaskia chichipe), para delimitar los terrenos, por sus frutos comestibles, es empleado como forraje, proporciona sombra para los seres humanos y el ganado, y es un espacio para el almacenamiento del forraje entre sus brazos o ramas. Otras especies preferidas pero menos abundantes en las parcelas son chende (Polaskia chende), con usos similares a los del chichipe; guaje (Leucaena esculenta) por sus vainas comestibles; el mezquite (Prosopis laevigata) que "llama al agua", proporciona sombra, leña y forraje; y el coahuino o pirul (Schinus molle) empleado como sombra, abono verde, medicina, combustible, para la elaboración de utensilios y como almacén de forraje entre sus ramas. Este sistema también proporciona el $75 \%$ de los requerimientos de frijol y maíz criollo y $50 \%$ del maíz requerido para el forraje en la comunidad (Moreno-Calles et al., 2012). En este sistema se desarrollan procesos de domesticación de especies de cactáceas columnares que son toleradas, protegidas y cultivadas en las parcelas debido a que proporcionan sombra, forraje, frutos comestibles y se emplean para almacenar el rastrojo. Además del chende y del chichipe, se incluyen el garam-

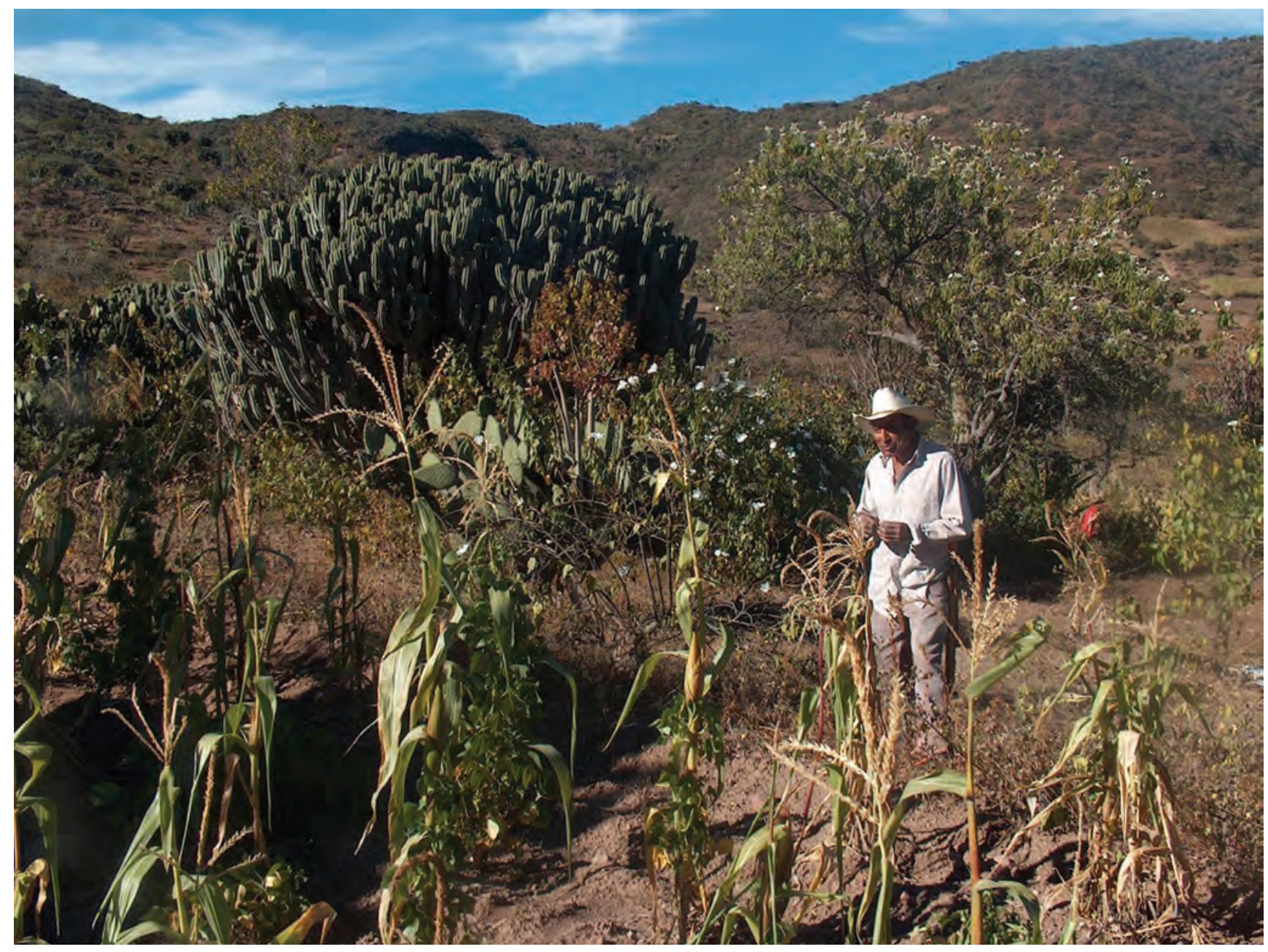

Figura 3. El sistema agroforestal de Milpa-chichipera en San Luis Atolotitlán en el Valle de Tehuacán Puebla. 
bullo verde (Myrtillocactus schenckii), jiotilla (Escontria chiotilla), xoconostli (Stenocereus stellatus) Riccob.) y pitaya de mayo (S. pruinosus), cuyas poblaciones manejadas en los SAF mantienen altos porcentajes (en promedio 94\%) de la diversidad genética que existen en poblaciones silvestres de estas especies (Blancas et al., 2006, 2009; Carmona y Casas, 2005; Casas et al., 2006; Otero-Arnaiz et al., 2003; Parra et al., 2008). La utilidad de las especies, su valor y las reglas de uso acordadas por las comunidades favorecen el mantenimiento de los SAF y de su diversidad biocultural. Procesos como la migración, el envejecimiento de las y los campesinos que los manejan, los cambios en el clima, la fragmentación de las parcelas agroforestales, los programas gubernamentales y la intensificación agrícola han incidido en la cobertura vegetal en el centro de las parcelas, lo que conlleva a la eliminación de las franjas contra la erosión y los árboles aislados.

El huamil, desarrollado en el Valle de Santiago, Guanajuato, involucra la apertura de áreas al cultivo dentro de los escurrimientos rocosos de los cerros (Palerm, 1997). Durante la roza se toleran a especies como garambullo (Myrtillocactus geometrizans) y pitaya (Stenocereus queretaroensis), que proporcionan frutos comestibles, así como mezquite (Prosopis laevigata), principalmente por su sombra (Colunga García-Marín et al., 1984). Los nopales y xoconostles en estos sitios son particularmente importantes y se pueden encontrar variedades toleradas, fomentadas y plantadas en los huamiles, así como en cercos vivos y huertos. Las variedades incluyen: hartón, cardón y sangre de toro (Opuntia streptacantha Lem.); lisita y sotaleña (O. hyptiacantha); apastillada silvestre (O. megacantha); xonocostle perón $(O$. lasiacantha); xoconostle amarillo, xoconostle barrilito, xoconostle cenizo, xoconostle colorado (O. joconostle); nopal blanco chico, nopal blanco grande y nopal blanco espada (O. atropes) (Colunga García-Marín 1984).

El territorio de Baja California Sur ha sido habitado desde hace aproximadamente 10,000 años por grupos indígenas (pericú, guaycura y cochimí), cuya estrategia de subsistencia se basaba en la caza, la pesca y la recolección (Cariño et al., 2012). La agricultura comenzó a practicarse en los siglos XVII y XVIII con la llegada de los misioneros jesuitas $\mathrm{y}$, posteriormente, de grupos de familias de Sonora, Sinaloa y Nayarit, las cuales crearon con las comunidades locales una cultura híbrida conocida como "rancheros". Los "rancheros" fueron llevados a la región para realizar las labores agrícolas rechazadas por los indígenas o porque muchos de los habitantes originarios murieron por enfermedades (Cariño et al., 2012). Con los "rancheros" se introdujo el ganado y la dominancia de las especies vegetales del Viejo Mundo a estos espacios (Nabhan et al., 2010). Un importante recurso que permitió el establecimiento de la agricultura en zonas áridas fueron los oasis, los cuales son fuentes naturales de agua permanente que brindan condiciones para el establecimiento de vegetación que en el área circundante no prospera y que además es el único tipo de agricultura que no requiere extraer agua del subsuelo para practicarse (Maya et al., 1997; Cariño et al., 2012). En la Península de Baja California, Maya et al. (1997) han identificado 184 oasis, casi todos en Baja California Sur. Los misioneros y los "rancheros" establecieron en los oasis huertas de frutales y hortalizas, de las cuales algunas sobreviven hasta el presente y constituyen verdaderos refugios culturales (Cariño et al., 2012). Uno de los primeros trabajos que evaluó el uso de recursos en los oasis es el de Breceda et al. (1997), los autores encontraron que el $47 \%$ de las especies de estos espacios son frutales, el $34 \%$ son hortalizas, $12 \%$ granos y $7 \%$ son especies de uso industrial. Nabhan et al. (2010) realizaron muestreos en nueve oasis en la Península de Baja California, y registraron el manejo de 57 especies perennes, principalmente frutales introducidos, aunque cabe resaltar que se desconocen a las especies que se encontraban en estos espacios antes del periodo colonial. Entre las especies más frecuentes se describen a las siguientes: aguacate fuerte (Persea americana), plátano (Musa $\times$ paradisiaca), limón real (Citrus limon), naranja dulce (C. sinensis), dátil (Phoenix dactylifera), higo prieto (Ficus carica), guayaba blanca y rosa (Psidium guajava), mango (Mangifera indica), granada temprana (Punica granatum), olivo misionero (Olea europaea) y ciruela amarilla (Spondias purpurea).

El kuojtakiloyan de la Sierra Norte de Puebla, el te lom de San Luis Potosí y el cacaotal en el Soconusco, Chiapas y la Chontalpa, Tabasco. Los agrobosques (bosques intermedios, bosques artificiales, bosques alterados, agroforests), son espacios donde los seres humanos han dirigido la composición de los árboles de acuerdo con sus necesidades, pero preservan características estructurales y procesos ecológicos que se desarrollan en los bosques considerados naturales (Alcorn, 1990; Wiersum, 2004).

Kuojtakiloyan significa en náhuatl "bosque que produce" o "bosque útil", es un sistema agroforestal donde hombres y mujeres de comunidades nahuas de la Sierra Norte de Puebla (Figura 4) manejan plantas silvestres y cultivadas y donde las características estructurales de los bosques originales son preservadas, aunque la composición de las especies ha sido modificada (Toledo y Moguel, 2012). En este sistema se cultiva café y se encuentra ubicado entre los 250 y los 1,000 $\mathrm{m}$ s.n.m., forma parte de un paisaje regional que incluye milpas, tierras en barbecho, tierras en pastoreo y remanentes de bosques templados y bosques mesófilos (Toledo y Moguel, 2012). La especies que brindan sombra para el café, y otras que se desarrollan en este sistema, dan como resultado una rica composición florística que incluye entre 250 y 300 especies de plantas, de las cuales el $96 \%$ son consideradas útiles, empleadas principalmente como alimento (125 spp.), medicinales (40 spp.), así como numerosas especies ornamentales, combustibles, melíferas y especies que son comercializadas como canela (Cinnamomum verum), ma- 


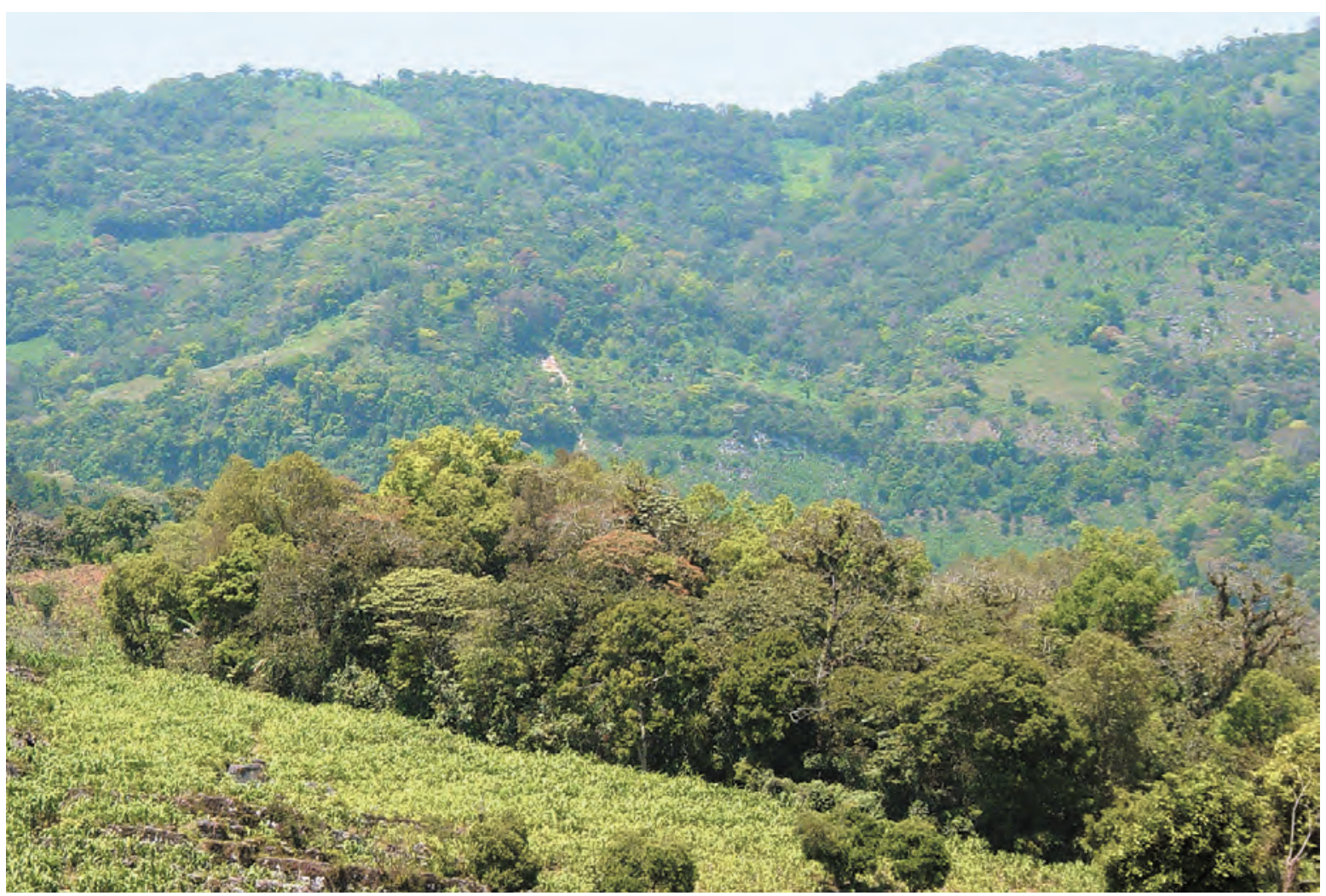

Figura 4. En la Sierra Norte de Puebla, predominan los mosaicos de paisaje que incluyen milpas, potreros, cañaverales y los sistemas agroforestales de café bajo sombra conocidos como Kuojtakiloyan, que parecen fragmentos de vegetación secundaria (acahuales), pero que en realidad son ensambles de especies útiles creados por la cultura náhuatl.

cadamia (Macadamia integrifolia), mango y café (Coffea arabica) (Moguel y Toledo, 1999, 2004; Toledo y Moguel, 2012). Varios de los productores son socios de la cooperativa Tosepan titataniske (unidos venceremos) que produce café orgánico para el mercado nacional e internacional. Además, proporcionan servicios turísticos ambientalmente amigables y benefician a los socios de la cooperativa con servicios de vivienda, salud y educación.

El te' lom es un SAF manejado por los teenek que viven en las zonas de bosque tropical perennifolio de San Luis Potosí y Veracruz. Este SAF puede parecer un bosque sin disturbio, pero la composición y distribución de las especies muestran el efecto del manejo humano. Puede contener elementos de vegetación primaria, bosque secundario y especies introducidas; te 'lom significa "grupos de árboles"(Alcorn, 1984). Este SAF generalmente se establece en zonas de pendiente pronunciada; brinda diversos recursos, así como sombra para el ganado o para el café. Son cinturones de vegetación alrededor de arroyuelos y manantiales para "evitar que se sequen", y espacios donde los niños juegan y las mujeres se bañan (Alcorn, 1983, 1984). El origen del te 'lom es dinámico y diverso; por ejemplo, un espacio que es manejado como milpa y que mantiene una gran cantidad de árboles útiles puede ser un espacio adecuado para establecer un télom. También lo son las tierras adjuntas a las casas abandona- das, donde generalmente existen numerosas especies útiles que pueden constituir el núcleo de especies originales para el télom. En el estudio realizado por Alcorn (1983), este SAF ocupaba una cuarta parte del área de la comunidad y se podían registrar alrededor de 300 especies de plantas, de las cuales se encontraban en la categorías de uso medicinal (221 spp.), consumo humano (81 spp.), para construcción (33 spp.), y otras 65 especies tenían usos diversos como la elaboración de utensilios y colorantes. En los manantiales, los árboles denominados ипир (Ceiba pentandra) son tolerados y plantados por considerarse como remedio para el sarampión y porque se piensa que protegen el agua superficial. Otras plantas son consumidas como frutos durante la estancia de las personas en el te lom o bien preparados en atoles, tales como el maap (Acrocomia mexicana), $h a$ ' pehte' (Eugenia oerstediana), tsabak (Mastichodrendron capiri) y maan'te (Pouteria campechiana). Las semillas de tiyow (Enterolobium cyclocarpum) son recolectadas y usadas para preparar una bebida parecida al café. Otras plantas que pueden ser encontradas en el te'lom por sus usos comestibles, medicinales, rituales y para obtener ingresos monetarios son: uh (aguacate, Persea americana), bolom it 'ath (mamey, Pouteria mammosa), tsab it'ath (Manilkara zapota), bek (guayaba, Psidium guajava), kukay (anona, Annona reticulata); tsatsa' (pitaya, Hylocereus undatus) y 
maango (mango, Mangifera indica). Se practica la remoción de árboles que no brindan algún uso, la de plantas que afectan la producción de otros recursos, o la de aquellos que están creciendo mucho y que, debido al viento, pueden caer y poner en peligro a otros recursos. Existen nombres para las prácticas de manejo de las plantas en teenek, las plantas pueden ser tumbadas (kwathal), dejadas o toleradas (jilath), limpiadas o desyerbadas ( $a k^{\prime i t h}$ ), cuidadas o protegidas (beletnath, k'anithach), transplantadas (tsaab táyath) o sembradas ( $t^{\prime}$ ayath), y las que hay en cualquier sitio o que están en el monte (alkith wa'ats). En los años ochenta, en el trabajo de Alcorn (1983), ya se identificaban importantes amenazas para este SAF. Los teenek en ese tiempo, y probablemente hasta la actualidad, eran exhortados por parte de instancias gubernamentales a hacer "uso" del te lom, pues desde la perspectiva gubernamental este sistema no es considerado productivo, como los pastizales, el cultivo de la caña de azúcar, los naranjales y la milpa. No existe mercado para los recursos que se obtienen del télom, lo que incentiva a los teenek a disminuir su área, situación paradójica, pues mientras los mangos, aguacates y otros frutos se caen de los árboles para pudrirse, las personas que han transformado sus tierras de agrobosques a pastizales tienen fuertes problemas para la obtención de frutos, madera, leña y materiales para construcción (Alcorn, 1983).

Otro tipo de agrobosque son los cacaotales, el nombre de cacao es una palabra aparentemente proveniente de la lengua olmeca kakawa, y que posteriormente fue usada por los mayas como kakaw, el término en lengua náhuatl derivada de esta palabra es cacahuatl (Coe y Coe, 1999). El cultivo y domesticación del cacao (Theobroma cacao) aparentemente fue iniciado por los olmecas en Mesoamérica, y desde tiempos precolombinos hasta la actualidad esta planta ha tenido un valor sagrado, ritual, social, medicinal, gastronómico, como tributo y como moneda de intercambio (Bergmann, 1969; Gómez-Pompa et al., 1990; Coe y Coe, 1999; Ogata, 2003; McNeil, 2009). El cacao fue cultivado casi en cualquier lugar que tuviera las condiciones necesarias de temperatura y humedad, desde Michoacán hasta Nicaragua en el oeste, y del norte de Veracruz a Honduras en el este. Incluso, se desarrollaron sistemas de irrigación donde no existían estas condiciones o bien se cultivaba en pozos subterráneos en el caso de Yucatán, pero de manera más importante fue cultivado en Tabasco, noreste de Oaxaca, en el centro y sureste de Veracruz, sureste de Chiapas y noreste de Guerrero (Bergmann, 1969; Gómez-Pompa et al., 1990; Rojas Rabiela, 1991; Young, 1994; Coe y Coe, 1999; Ogata, 2003). Los mayas son considerados los principales diseñadores de un sistema agroforestal de cacao muy sofisticado, que incluía el manejo de árboles de sombra, riego, viveros y técnicas de transplante (Ogata, 2003). Los mexicas también tuvieron conocimiento del manejo de esta planta y en la obra de Hernández ([1790]1942) sobre la "Historia de plantas de la Nueva España" se relata el cultivo del cacahoaquahuitl (árbol de cacao), donde se distinguen cuatro tipos diferentes de esta planta y el manejo de especies de sombra asociadas conocidas como cacahuanantli o madres del cacao. Entre estas plantas destaca el atlinam (Licaria arborea), llamada también cacahuanantzin, cacahuananche o madre del cacao. La característica del cacaotero, de requerir la sombra de otros árboles, está relacionada con condiciones de humedad y sombra específicas para las plantas, así como para los insectos que polinizan sus flores (Young, 1994; Coe y Coe, 1999). Actualmente los cacaotales en el Soconusco y la Chontalpa constituyen ejemplos notables de bosques manejados, donde además del cacao, se mantiene un elevado número de especies vegetales y animales domesticados y silvestres, de especies nativas e introducidas, lo cual imita un bosque pero que en realidad tiene una alta dominancia de especies útiles en su composición (Ogata, 2003). Se han desarrollado estudios que registran la diversidad de especies de árboles cultivados y manejados en los cacaotales de Chiapas y Tabasco para proporcionar sombra, principalmente como especies maderables o frutales, se estiman alrededor de 20 especies, entre las que destacan las siguientes (Magaña, 2006; Salgado-Mora et al., 2007): cocoíte (Gliricidia sepium), empleada como cerco vivo y medicinal; pataxte (Theobroma bicolor), empleado para elaborar bebidas parecidas al chocolate; samán (Pithecellobium saman), ornamental; mango (Mangifera indica), con frutos comestibles y madera aprovechable; moté (Erythrina americana); amate (Ficus padifolia); palo mulato (Bursera simaruba), usada como cerco vivo; cedro (Cedrela odorata), una madera de excelente calidad; quijinicuil (Inga jinicuil), sus frutos son comestibles; aguacate (Persea americana), cuyos frutos son comestibles; achiote (Bixa orellana), con frutos comestibles y colorante; hule (Castilla elástica), se emplea su látex, su madera y es medicinal; zapote (Pouteria sapota), sus frutos son comestibles; guácimo (Guazuma ulmifolia), empleado como cercos vivos y leña; pimienta (Pimenta dioica), una especia y medicinal; anona (Annona reticulata), frutal y medicinal; y cítricos (Citrus spp.).

El metepantli, metepantle o metepancle de Tlaxcala. Las tierras de laderas pronunciadas, las zonas secas con largas estaciones sin precipitación y bajas temperaturas en algunas épocas del año, son algunos rasgos predominantes en los escenarios agrícolas de México (Rojas Rabiela, 1991). En este contexto, la lucha por mantener el suelo, la fertilidad, la humedad y disminuir el efecto de las heladas en los sistemas agrícolas fue y es una de las preocupaciones constantes para los campesinos (Wilken, 1987). Una de las formas de resolver tales problemas ha sido el desarrollo de sistemas de terrazas de los cuales se han encontrado registros con una antigüedad de entre 3,000 y 2,000 años en el Centro de México, el Valle de Tehuacán y Oaxaca (Donkin, 1979). Estos sistemas también se han registrado en distintos periodos en el noroeste de Chihuahua, el Valle de Sonora, la 
Sierra de Tamaulipas, la Cuenca de México, la Cuenca de Toluca, el estado de Hidalgo, el Valle del Río Chilchota, en Michoacán, el Valle Poblano-Tlaxcalteca, la Mixteca Alta, y el Suroeste de México. Tan relevantes y ampliamente distribuidos en Mesoamérica son estos sistemas, que han sido sugeridos por varios autores como uno de los elementos para definir el área biocultural regional (Donkin, 1979; Rojas Rabiela, 1991; Whitmore y Turner II, 2001). En México se reconocen tres tipos de terrazas: (1) tenamitl (muro), tecinta, terraza de ladera, besana, cerca, flat terrace o bench terrace, en la cual el muro o retén de la terraza es de piedra, tepetate o tierra; son construidas en laderas escarpadas con la finalidad de retener sedimentos y humedad; (2) presa, trinchera, atajadizo, lama y bordo, tecercas o check dams, las cuales se construyen en el interior de barrancas, cárcavas o pequeños arroyos, son muros de piedra o represas en los que se acumulan sedimentos y humedad; y (3) los metepantlis, terrazas en laderas suaves, bancales, melgas, semiterrazas o slope terraces, las cuales se construyen en laderas de pendiente suave, modificando levemente la superficie con el levantamiento de bordos que son estabilizados con plantas, con la finalidad de reducir la erosión y absorber la humedad (Rojas Rabiela, 1991). En Tlaxcala se desarrolla una práctica agroforestal conocida como metepantli, metepan- tle o metepancle (Figura 5), ésta es una palabra que deriva del náhuatl metl, que significa "maguey", y pantli "espacio entre" (Whitmore y Turner II, 2001), aunque también se discute si el significado de metepantli corresponde a "pared de magueyes" (González-Jácome, 2003). Los campesinos de Tlaxcala suelen hacer referencia a los pancles o pantles como las zonas cultivables de un bancal, y se refieren a metepancles o metepantles a los bordos de tierra que dividen dicha superficie y que contienen una serie de plantas como los magueyes y frutales (González-Jácome, 2003). En entrevistas realizadas recientemente, hemos encontrado que los campesinos de Tlaxcala también llaman metepantles a todo el conjunto del sistema donde se desarrollan las prácticas mencionadas. Entre las especies más utilizadas se han descrito al maguey manso (Agave salmiana), aunque pueden existir otras especies de magueyes (Agave spp.), nopales (Opuntia spp.), sabino (Juniperus deppeana), tepozán ó toposán (Buddleja americana), encino (Quercus castanea), pino (Pinus leiophylla); árboles frutales nativos como tejocote (Crataegus mexicana) y capulín (Prunus capuli), así como especies introducidas como durazno (Prunus persica) y chabacano (Prunus armeniaca); además de especies de arbustos como palo dulce (Eysenhardtia polystachya) y jarilla (Senecio salignus) (Donkin, 1979; Altieri y Tru-

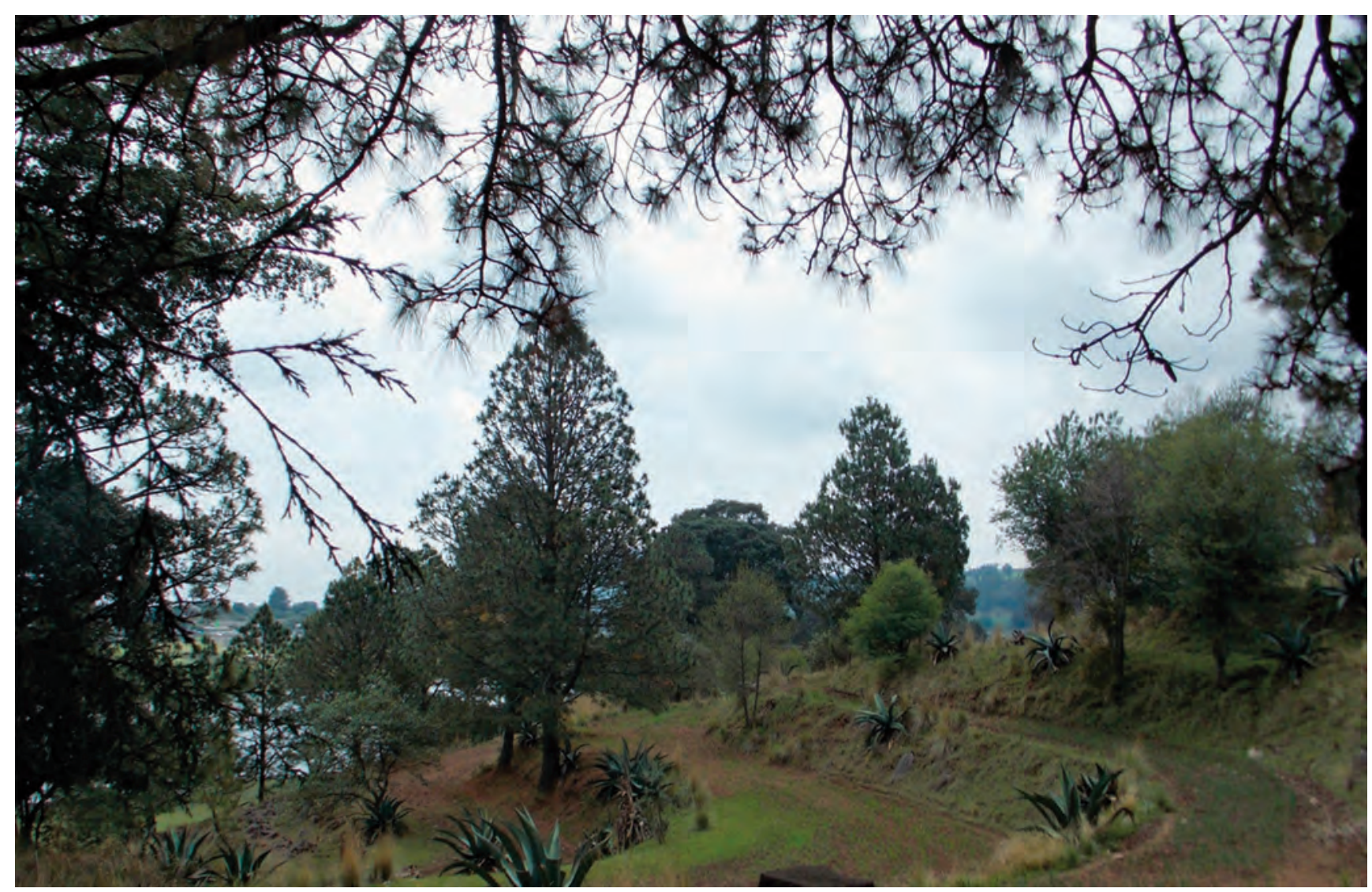

Figura 5. El metepantle en San Felipe Hidalgo Nanacamilpa, Tlaxcala. 
jillo, 1987; Wilken, 1987; Mountjoy y Gliessman, 1988; Evans, 1990; Rojas Rabiela, 1991; Whitmore y Turner II, 2001; González-Jácome, 2003; Magdaleno et al., 2005). Estos árboles y arbustos generalmente son resistentes a la sequía y tienen importantes funciones; por ejemplo, los $m a$ gueyes son empleados por sus amplias raíces superficiales y aglutinantes, pues la función del metepantle es prevenir la erosión y acumular humedad. Los magueyes represan y colectan agua de lluvia, ayudan a la escorrentía, mientras que sus hojas se emplean como abono y combustible (Altieri y Trujillo, 1987). Las diversas especies de magueyes que se encuentran en los metepantles tienen múltiples usos: (1) preparación de bebidas como aguamiel, pulque y el mezcal; (2) hábitat de especies valoradas local y regionalmente, y que son consumidas y comercializadas, como los gusanos blancos de maguey que se producen en temporada de secas, los gusanos rojos conocidos como chiloquitl, y los gusanos amarillos que llevan de nombre nixtamal; (3) fibras para elaborar lazos, collares y sostenes de los animales; (4) utensilios como agujas, elaboradas a partir de sus espinas, y mixiotes para sostener la carne que se prepara con este nombre; (6) comestible, por sus flores que son consumidas como verduras y sus tallos cocidos denominados mezcal; (6) en la construcción, pues sus quiotes se utilizan como vigas y postes; (7) para la obtención de un tipo de material combustible a partir del maguey seco llamada mesontete (Donkin, 1979; Altieri y Trujillo, 1987; Wilken, 1987; Mountjoy y Gliessman, 1988; Evans, 1990; Rojas Rabiela, 1991; Whitmore y Turner II, 2001; Magdaleno et al., 2005; González-Jácome, 2011).

El sabino es una especie de uso ritual muy importante en Tlaxcala; las personas de la comunidad de Tlaxco, Tlaxcala, eligen un árbol de esta especie para que cuando mueran les hagan una cruz con éste, cuya madera es durable y de buena calidad, el sabino además es valioso como sombra y para marcar con sus tocones secos los límites entre localidades (mojoneras). Esta especie es tan valiosa localmente que si se llega a talar un árbol por algún miembro de la comunidad sin que éste haya sido autorizado por la asamblea, será sancionado con el pago de 10,000 pesos (769 dólares). Los árboles y arbustos como nopales, tejocotes, capulines y $d u$ raznos son valorados principalmente por sus frutos y semillas comestibles. En el caso de los capulines, los campesinos distinguen dos clases, los de las semillas suaves y pequeñas, las cuales son asadas, saladas y comercializadas, y los de frutos grandes que son los preferidos para ser consumidos como fruta de temporada y para el dulce de capulín (Altieri y Trujillo, 1987; Wilken, 1987; Mountjoy y Gliessman, 1988; Evans, 1990; Rojas Rabiela, 1991). En el nopal, los campesinos distinguen cuatro tipos diferentes, de los cuales no se han identificado sus especies, estos son el nopal tuna tapona, nopal cimarrón, xocayontle y nopal cultivado.

Entre los principales problemas que enfrenta el metepantle destacan: (1) la pérdida de magueyes debida a la extracción clandestina de mixiote, lo que hace al maguey vulnerable y que en poco tiempo muera; ello desincentiva a las personas que los plantan, pues deben esperar ocho años para aprovechar los magueyes; (2) la pérdida de las prácticas de manejo del maguey en los metepantles debido a la disminución del número de personas en las comunidades por migración o porque las personas que permanecen no están dispuestas a invertir la gran cantidad de tiempo y esfuerzo que representa esta actividad; y (3) el empleo de tractores en las actividades agrícolas donde otrora no se utilizaban, lo que requiere la eliminación de los bordos, y de las plantas en ellos, para facilitar la labor de esta maquinaria.

La chinampa en el Valle de México y el calal del suroeste de Tlaxcala. En los lagos, orillas de los ríos y/o pantanos del Valle de México (West y Armillas, 1950), del Valle de Toluca (West y Armillas, 1950; Albores 1995), del suroeste de Tlaxcala (Wilken, 1969;García-Cook, 1974) y en la Península de Yucatán (Siemens y Puleston, 1972), hace aproximadamente 2,000 años se desarrolló una agricultura intensiva, con pocos o ningún periodo de descanso, brindando cosechas continuas con altos niveles de productividad. Son sistemas en los que el suelo es elevado con respecto al nivel del agua para drenarlos, utilizando para ello materiales como lodo, materia orgánica, árboles, conglomerados de vegetación, entro otros materiales, con el fin de estabilizar una porción de tierra a manera de islote, y drenados por medio de canales. Tales sistemas son conocidos en la literatura como agricultura de campos elevados (raised fields) o agricultura de campos drenados (drained fields). Sobreviven pocos sistemas de este tipo en la actualidad, en México los más conocidos son las chinampas del Valle de México y los camellones o calales del suroeste de Tlaxcala.

Las chinampas son parcelas artificiales de forma rectangular con islotes largos y angostos, cuyas proporciones permiten la captura de humedad de las zanjas o canales chinamperos (Rojas Rabiela et al., 2009). Se piensa que las chinampas fueron construidas "a mano" sobre entretejidos de vegetación acuática natural conocidos como céspedes, atapalacatl o pantano, apilando sobre estos lodo del lago, tierra y otros conglomerados (Rojas Rabiela, 1993; Cervantes y Rojas Rabiela, 2000). Los conglomerados incluían a algunas especies de tule (Scirpus lacustris y Typha angustifolia), además de otras especies presentes en el lago. Las plantas de tule han sido utilizadas para elaborar abrigos o petates, para cubrir los almácigos y como alimento, fertilizante, medicinas y forraje (Lot et al., 1979). En la actualidad, el lirio acuático o césped (Eichhornia crassipes), planta introducida, está siendo empleada como abono verde aplicado a los suelos de las chinampas y como forraje (Quiroz-Flores et al., 2008).

Una de las características más conspicuas del paisaje chinampero son los ahuejotes (Salix bonplandiana), árboles de rápido crecimiento y poca sombra que son sembrados en 
las orillas de los islotes, chinampa deriva de la palabra náhuatl chinamitl que significa "seto, nido o cerca de cañas" (Jiménez-Osornio y Gómez-Pompa, 1987), nombre con el que se refieren los agricultores al entretejido de troncos y varas de los ahuejotes con los que se refuerzan a las parcelas artificiales (Rojas Rabiela et al., 2009). Los ahuejotes tienen importantes funciones para la retención de la tierra, pero también se emplean como material de construcción y combustible, sus ramas sirven para cubrir los almácigos en invierno y para construcción de utensilios como canastas, se emplean como cortina contra el viento y para romper los patrones de movimiento de los insectos considerados plagas (Jiménez-Osornio y Gómez Pompa, 1987; Frederick, 2007). También se han empleado como soporte para plantas trepadoras como los chayotes y los frijoles (Rojas Rabiela, 1993; Sanders, 1993).

Las chinampas han sido de gran interés académico puesto que son considerados de los sistemas más intensivos del México prehispánico que perviven hasta nuestros días (Rojas Rabiela, 1993). Estos sistemas agroforestales son extraordinariamente fértiles y productivos debido al suelo orgánico que los compone, lo que les permitió albergar una gran cantidad de cultivos que han sido capaces de abastecer una elevada población (Armillas, 1971; Rojas-Rabiela y Sanders, 1985; Jiménez-Osornio y Gómez Pompa, 1987; Rojas Rabiela, 1993; Sanders, 1993). Entre los cultivos de la época prehispánica que se desarrollaban en las chinampas se describen maíz (Zea mays), uauhtli o amaranto (Amaranthus cruentus), jitomate (Lycopersicon esculentum), frijol (Phaseolus vulgaris), tomate (Physalis philadelphica), calabaza (Cucurbita pepo), chía (Salvia hispanica), quelites (Amaranthus spp., Chenopodium spp., Porophyllum spp.), chayote (Sechium edule) y chilacayote (Cucurbita ficifolia), diversas flores de ornato y hierbas de olor; posteriormente durante el período colonial, se incorporaron a las plantas cultivadas de las parcelas lacustres a hortalizas como pepino (Cucumis sativus), coliflor (Brassica oleracea var. botrytis), col (B. oleracea var. viridis), brócoli (B. oleracea var. italica), alcachofa (Cynara scolymus), ajo (Allium sativum), cebolla (Allium cepa), espinaca (Spinacia oleracea), betabel (Beta vulgaris), acelga (B. vulgaris var. cicla), cilantro (Coriandrum sativum), perejil (Petroselinum crispum), apio (Apium graveolens), rábano (Raphanus sativus), poro (Allium porrum), zanahoria (Daucus carota), además de hierbas de olor como romero (Rosmarinus officinalis) y albahaca (Ocimum basilicum). Quedan también hasta la actualidad algunos árboles nativos como tejocote y varios árboles introducidos como durazno, membrillo (Cydonia oblonga) y chabacano (Prunus armeniaca) (Rojas Rabiela 1993).

Existen varías problemáticas a las que se enfrenta la agricultura chinampera, como el incremento del lirio acuático, lo que ocasiona problemas ecológicos; la salinización de las chinampas es un problema importante debido al deseca- miento de los lagos y canales, a la eliminación de los árboles, a la pérdida de prácticas de reposición de los mismos y disminución de la cobertura vegetal, así como el incremento de agroquímicos y de invernaderos en estos sitios. La urbanización de estas zonas es otro proceso que amenaza la permanencia de estos sistemas (Stephan-Otto, 2005; Merlín-Uribe, 2013).

Calal significa en náhuatl "casa junto al agua", es un sistema agroforestal de gran antigüedad en Tlaxcala, distribuido en el suroeste del estado (Figura 6) (González-Jácome, 1999; 2008), aparentemente precolombino (Wilken, 1969, 1987; Denevan, 1970; Crews y Gliessman, 1991). Se desarrolla actualmente en las llamadas tierras de jugo o de humedad por los campesinos tlaxcaltecas, en un contexto de suelos pobremente drenados, con aguas subterráneas a niveles altos y lluvias variables en el verano (Wilken, 1969, 1987). El sistema de calal combina franjas angostas de tierra rodeadas por los cuatro lados con zanjas o canales de drenaje; la construcción de estos canales implica la excavación del suelo, el cual es colocado sobre la tierra entre los canales, dando como resultado plataformas elevadas para el cultivo y una matriz de canales en el paisaje (Crews y Gliessman, 1991). Para estabilizar los canales e incrementar el drenaje se plantan en las orillas árboles que proporcionan nitrógeno al sistema. Entre las especies más importantes se pueden mencionar el aile (Alnus firmifolia), el ahuejote ( $\mathrm{Sa}$ lix bonplandiana), los frutales tejocote y capulín. Arbustos como asomiate (Senecio salignus) y jarilla (Baccharis glutinosa) son empleados como combustible para los temazcales y fogones (Wilken, 1969). González-Jácome (2008) describe la tolerancia de árboles en los canales mayores, principalmente Populus nigra y Alnus sp., y observó que los canales de menor tamaño eran arbolados y se practicaban actividades de poda para impedir que tuvieran demasiada sombra sobre las plataformas; las especies manejadas de esta manera incluyen a tejocote, capulín, álamo blanco (Populus alba), especies de aile (Alnus spp.) y fresno (Fraxinus uhdei). Actualmente los canales pequeños están siendo rellenados para incrementar el área cultivable y poder hacer uso de maquinaria y camiones de carga para las actividades agrícolas, de tal manera que los árboles en estos lugares están desapareciendo. Como en el caso de las chinampas, la vegetación acuática también es importante en estos sistemas, entre éstas son los berros (Hydrocotyle ranunculoides y Nasturtium officinale) y la palmita de agua (Berula erecta), que es medicinal y consumida como quelite (González-Jácome, 2008). Así también, son utilizadas especies de tule para la elaboración de petates, tenates y sombreros, y como fuente de materia orgánica que se almacena en los canales (Crews y Gliessman, 1991). También para la alimentación de animales domésticos se utilizan plantas tales como acacapa (Eupatorium morifolium), acahuale (Tithonia tubaeformis), acatextle (Sagittaria latifolia), achochote (Bidens pilosa), altatana (Cucurbita sp) y nixtule esquina- 


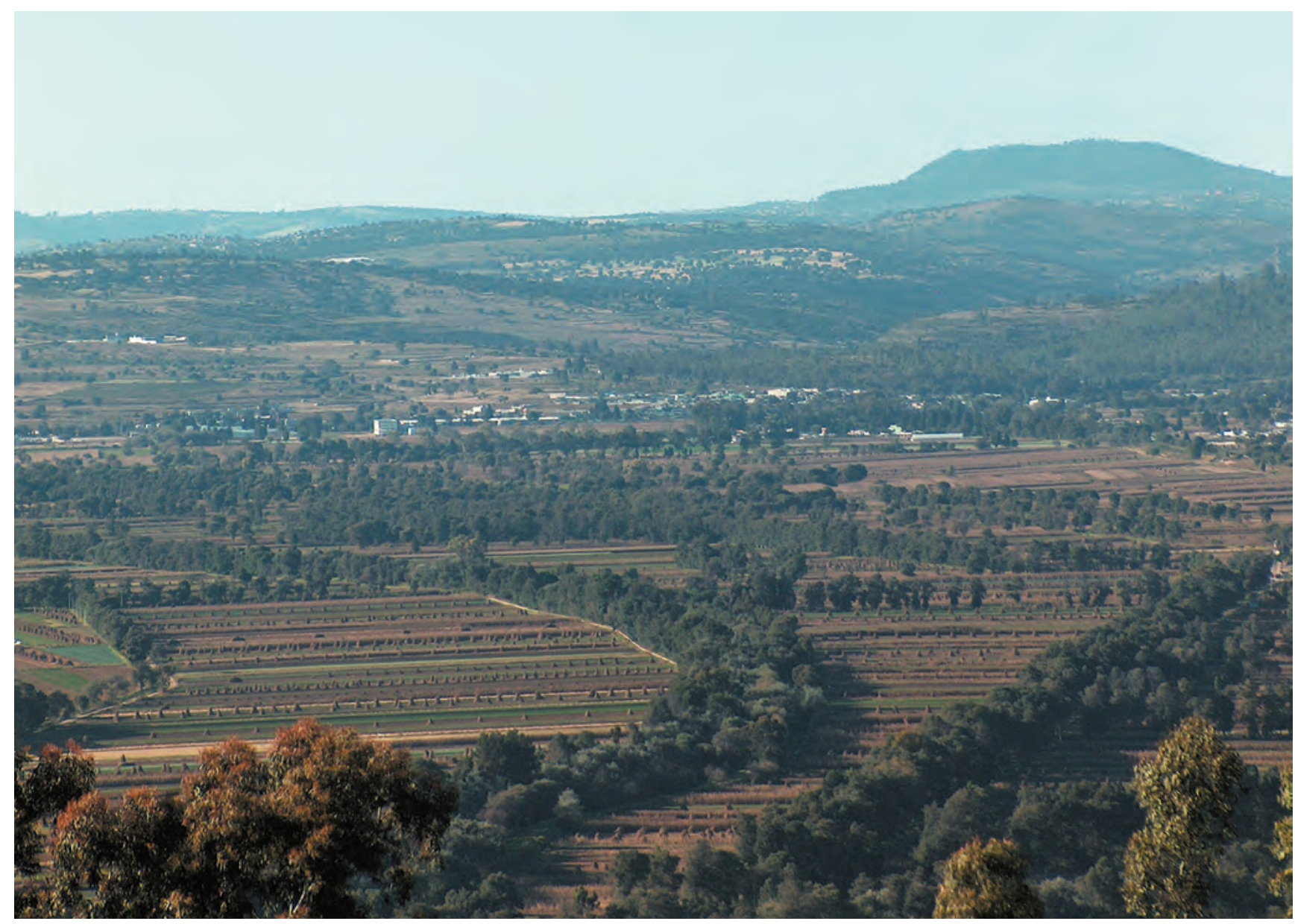

Figura 6. El calal en el suroeste de Tlaxcala.

do y nixtule redondo (Eleocharis spp.; González-Jácome, 1999, 2008). Es un sistema que está modificándose y extinguiéndose rápidamente debido a procesos como contaminación del aire, suelo y vegetación, plagas, y falta de fuerza de trabajo para mantener el sistema, la pérdida del conocimiento y prácticas tradicionales de manejo, las disputas por el control político y económico del suelo y agua que se dan entre campesinos, gobierno y empresas constructoras (González-Jácome, 2008).

El huerto, solar, patio y ekuaro. El concepto de huerto, solar, homegarden, dooryard garden, house lot garden, calmil (náhuatl), ekuaro (purhépecha), wal eleb (teenek), kaj ka'a (chontal) y patxoconna (maya), es un sistema agroforestal que se encuentra cerca o al lado de la casa, donde se manejan múltiples especies perennes y anuales y en algunas ocasiones animales (Fernandes y Nair, 1986; Kumar y Nair, 2004; Montagnini, 2006). Existen varias hipótesis acerca del surgimiento de estos espacios cuya característica principal es su ubicación al lado de las habitaciones humanas, y podría considerarse uno de los sistemas agroforestales más antiguos en México: En el Valle de Tehuacán, MacNeish
(1967) propone que hace aproximadamente unos 5,000 años se desarrolló la "horticultura de barranca", la cual define el autor como la siembra de cultivares resistentes (calabazas) en las barrancas que se encontraban cercanas a las cuevas habitadas por seres humanos. En la región de la Península de Yucatán, se piensa que algunas de las primeras formas de huerto en esta región pudieron provenir del aclareo de la vegetación para el establecimiento de las habitaciones humanas; a partir de estas perturbaciones, se incrementaron algunas especies vegetales útiles, pero también se favoreció la atracción de especies de animales que podían cazarse, esta hipótesis es conocida como "hunting-garden" (Linares, 1976). Doolittle, (2004) emplea el término "exaptations", para explicar el origen de los huertos; el autor explica que estos sistemas pudieron ser inicialmente espacios cercanos a las casas donde se depositaban los desechos humanos tales como semillas o partes de plantas, sin que inicialmente esa fuera la intención humana, con el tiempo estos espacios se volvieron muy importantes para suministrar recursos útiles de plantas en cercanía a las habitaciones humanas. En las zonas altas, la construcción de terrazas favorecían la defensa de los territorios por la visibilidad y la dificultad de 
ascenso a ellas, lo que fomentaba su construcción para el establecimiento de viviendas y zonas de cultivo, las cuales eran llamadas en náhuatl calmil (Evans, 1990). Trabajos recientes proponen que el huerto, como lo conocemos actualmente, surge a partir de la fusión del huerto (sistema de árboles frutales) y la huerta (sistema destinado a la producción de legumbres y vegetales) en una sola unidad, incorporando en la época colonial a especies exóticas de frutales y ganado mayor (González-Jácome, 2011). Las diferencias entre huerta y huerto tienen relación con la escala y no necesariamente con la composición, donde huerta se refiere al sitio grande y huerto al sitio pequeño, la autora describe que en las fuentes del siglo XVI consultadas por ella, incluyen más frecuentemente el uso del término huerta tanto para los sistemas dominados por especies perennes casi a nivel de monocultivo, como en el sentido de "huerta de cacao", y también a las huertas de perennes y anuales donde podemos ubicar a los huertos domésticos (Rojas-Rabiela com. pers.)

La estructura y composición de los huertos actuales en Mesoamérica es compleja, conteniendo varios estratos horizontales y verticales, pudiendo ser dominantes las especies perennes emulando bosques, hasta una estructura más cercana a la de los campos de cultivo con las especies perennes ubicadas de manera intersticial y como cercos vivos, y con una dominancia de especies arbustivas y herbáceas (Montagnini, 2006). Estos sistemas son valorados en su contribución a la diversidad biocultural debido a: (1) se mantiene una gran cantidad de plantas nativas y exóticas útiles resultantes de la historia cultural de los pueblos que los manejan, (2) son sitios donde se desarrollan procesos de experimentación y domesticación de plantas antes de llevarse a los campos de cultivo, (3) se desarrollan experiencias de manejo de las especies y variedades en estos espacios ,y (4) proveen de materiales y son el escenario de actividades rituales (Niñez, 1987; Montagnini, 2006). Caballero et al. (2010) hicieron una revisión de información en la que registran 1,400 especies de plantas utilizadas en los huertos de México. Estos autores documentan 537 especies de plantas herbáceas, 224 de arbustos y 228 de árboles; de las cuales 572 especies tienen uso medicinal, 528 son ornamentales, 442 comestibles y 682 tienen otros usos.

Los huertos existen en nuestro país en distintos contextos culturales y ecológicos. Para las zonas templadas se describen ejemplos notables como el ekuaro, el cual se desarrolla en el estado de Michoacán por los pueblos purhépechas de las Cuencas del Lago de Pátzcuaro y Zirahuén, de la Meseta Purhépecha y por comunidades mestizas asociadas a éstos. El ekuaro es un espacio productivo manejado principalmente por mujeres y niños, y se encuentra inserto en una estrategia local de subsistencia que incluye la recolección y el manejo del bosque, el cultivo de parcelas agrícolas y el aprovechamiento de recursos lacustres (Mapes et al., 1990; Alarcón-Chaires, 2001). Los solares de las partes altas de la Cuenca del Lago de Pátzcuaro son muy relevantes para el cultivo de las variedades de maíz nativo de color rojo (tsiri charhápiti) y azul (tsiri tsirangui). Estos maíces han crecido por largo tiempo en los ekuaros y prefieren estos espacios donde las fuentes de nitrógeno y materia orgánica son más altas debido a la aportación de desechos vegetales y excrementos de animales presentes en el huerto. Además de los maíces, las mujeres hasta hace algunos años lograban mantener en el ekuaro a cultivos antiguos como el frijol kókok (Phaseolus coccineus; Mapes et al., 1990). En la Meseta Purhépecha, en la comunidad de Nahuatzen, Alarcón-Chaires (2001) describió el manejo de alrededor de 50 especies de plantas y animales en $300 \mathrm{~m}^{2}$, los principales usos de las plantas incluyen a la alimentación (66\%), donde se encuentran el maíz, principalmente de variedades nativas; los nopales y los frutales, las plantas medicinales(30\%) y otros usos que incluyen a las plantas ornamentales.

En Candelaria Loxicha, zona subcálido- húmeda donde habitan pueblos zapotecos, Aguilar-Støen et al. (2009) documentaron que las personas distinguen a los huertos de los patios, consideran a los primeros como espacios para la producción para el mercado y a los segundos como espacios para numerosas funciones, más semejante al concepto de huerto planteado en este trabajo. Los patios son importantes reservorios de diversidad de plantas útiles, los autores registraron 233 especies en 31 patios, donde dominan los árboles con usos comestible y ornamental, pero también plantas medicinales, leña, especias, artesanías y construcción. Un uso interesante descrito por los autores es el de "lujo", el cual es una expresión asociada al mantenimiento de una planta sin ningún otro uso que el disfrute de la misma. Este espacio es también un importante escenario de experimentación de las especies que se introducen en las estrategias locales de subsistencia; por ejemplo, cuando el café llegó a la región, las semillas y las plántulas fueron plantadas primero en los patios para aprender cómo cuidar a la nueva planta. Fue así también con la vainilla (Vanilla mexicana), de tal manera que los campesinos llevaron las plantas de vainilla desde el bosque a los patios y colocaron éstas en las ramas de distintos árboles, a diferentes alturas y con distinta sombra para probar el mejor espacio para el desarrollo de la planta. Lo hacen también con las plantas exóticas que pueden conseguir cuando viajan a otros lugares.

En Yucatán, el huerto maya es un área anexa a la casa con árboles: arbustos y hierbas que son seleccionadas para la producción de frutos, plantas medicinales y ornamentales, de manejo de animales y también es una fuente de ingresos monetarios (Rico-Gray et al., 1990). Este sistema junto con el de roza, tumba y quema constituyen dos pilares relevantes en la estrategia de subsistencia local (Gillespie et al., 2004). De acuerdo con Gómez-Pompa et al. (1987) y Mariaca-Méndez et al. (2010), los antiguos mayas delimitaron grandes áreas en las que se sembraron especies vegetales con usos múltiples, en las que además de tener una albarrada (muro de piedra) tuvieron árboles en la periferia (cercas 
vivas). Este sistema es conocido hasta la actualidad como petkot. Aparentemente una de las funciones principales del petkot era atraer especies de animales silvestres para cazarlos más fácilmente (Gómez-Pompa et al., 1987; MariacaMéndez et al., 2010). En la actualidad las cercas vivas son comunes en los huertos mayas yucatecos, en los caminos blancos (sacbé) y en las milpas de cultivo, y además de la delimitación de los terrenos; tiene funciones importantes como suministro de bienes materiales, resinas, medicinas, forraje, plantas para sombra y para techo, abonos verdes y como plantas fijadoras de nitrógeno (Flores et al., 2010). En su trabajo, Flores et al. (2010) narran los pensamientos de un campesino maya llamado Escolástico, con relación a los petkot: "los aluxes cuidan los campos de cultivo y los huertos, y mientras en el monte tienen que moverse entre muchos árboles para cuidarlos, en las cercas de huertos y milpas cuidan a los árboles uno por uno por la importancia que tienen para los hombres, los aluxes les enseñaron a los seres humanos a usar las plantas y a seleccionar las que debían estar en las cercas". Otro trabajo clásico es el realizado en las comunidades de Tixpehual y Tixcacaltuyub, donde Rico-Gray et al. (1990) encontraron alrededor de 135 especies de plantas, de las cuales el $60 \%$ de las más abundantes son nativas a la Península de Yucatán y la gran mayoría eran empleadas para la obtención de frutos comestibles y como plantas ornamentales, aunque también se describen usos rituales, medicinales y como madera.

Para las zonas áridas y semiáridas destacan los estudios de huertos de Blanckaert et al. (2004) en San Rafael Coxcatlán, en el Valle de Tehuacán-Cuicatlán. Los autores describen una riqueza de 233 especies que incluyen árboles, arbustos y herbáceas en 30 huertos estudiados, $66 \%$ de las especies tienen uso ornamental, $30 \%$ son comestible, $15 \%$ brindan sombra y $8 \%$ son medicinales. La mayoría de las plantas en estos espacios son cultivadas (70\%), principalmente las ornamentales, el resto son plantas toleradas y en algunos casos protegidas (Figura 7).

\section{Discusión}

Los sistemas agroforestales tradicionales de México: ubicación, pueblos que los manejan, contexto ambiental en el que se desarrollan. En la presente revisión se documentaron 20 nombres de sistemas agroforestales actualmente en uso, principalmente ubicados en las porciones centro y sur del país; destacan la baja presencia de estos sistemas en el norte, lo anterior puede estar relacionado porque efectivamente no existan sistemas agroforestales tradicionales en esta parte del país, bien porque no se han realizado los estudios correspondientes o por el alcance de esta revisión. Se identificaron vacíos de información acerca de los pueblos originarios que los manejan; esta revisión solo presenta ejemplos para ocho de estos grupos (nahuas, mepha's, ñiusavi, mayas, ñañu, teenek, purhépecha y zapotecos) de los 65 registrados en el
Atlas de Pueblos Indígenas de México publicado por la Comisión de Desarrollo de los Pueblos Indígenas (CDI, 2012), además deben considerarse también a los diversos grupos mestizos. Es necesario profundizar esta revisión en las etnografías de los distintos grupos de pueblos originarios para identificar otros grupos y regiones en las cuales se practican. La construcción de una base de datos en esta dirección permitirá un importante diagnóstico e inventario de conocimientos y técnicas para diseñar estrategias nacionales que ayuden preservar tan importantes sistemas de producción. Asimismo, es interesante destacar que bajo las distintas condiciones ambientales (templada, cálido-húmeda, árida y semiárida, y humedales) fue posible encontrar registro de sistemas agroforestales tradicionales, destacando que los sistemas intensivos de casa, conocidos genéricamente como huertos, se han registrado en todas las condiciones ambientales, mientras los demás tipos de sistemas corresponden al parecer a condiciones ambientales específicas.

Satisfacción de necesidades locales y globales. Las comparaciones de los SAF revisados con los sistemas forestales silvestres y con los sistemas agrícolas convencionales nos permite tener una aproximación de su contribución a la satisfacción de las necesidades locales y globales. Con base en diversos estudios florísticos y etnobotánicos en distintas

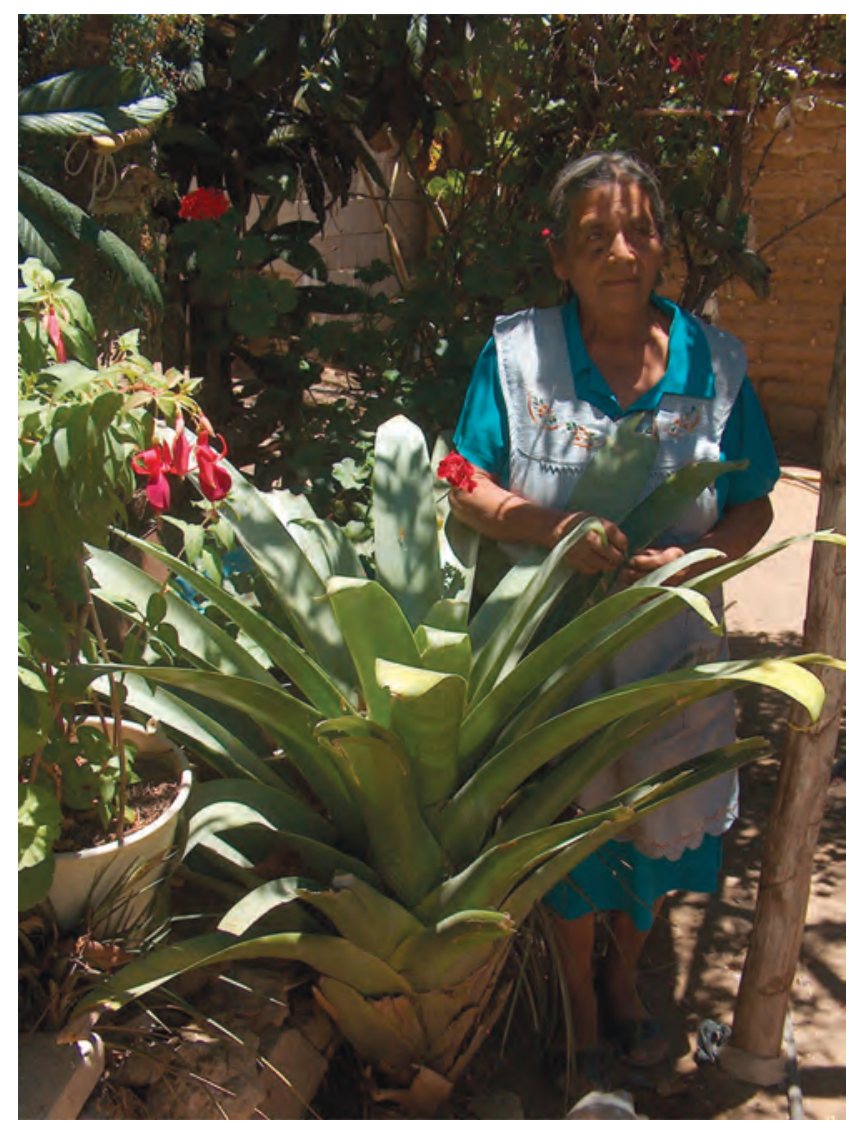

Figura 7. El solar en la porción semiárida del Valle de Tehuacán. 
regiones bioculturales del país, Sierra de Manantlán (Benz et al., 1994; Vázquez-García et al., 2004), Selva Lacandona, Los Tuxtlas, Tuxtepec, Uxpanapa y Sian Ka'an (Toledo et al., 1995), Península de Yucatán (Rzedowski, 1993; FloresGuido, 1998), Valle de Tehuacán (Dávila et al., 2002; Lira et al., 2009), zonas áridas y semiáridas del país (Rzedowski, 1993; Casas et al., 2010), Montaña de Guerrero (Casas et al., 1994), es posible estimar que en promedio el $39 \%$ de las especies encontradas en las bosques, selvas y matorrales tienen algún tipo de uso (Moreno-Calles, 2010). No obstante que aún es escasa la información al respecto para la mayoría de los sistemas agroforestales revisados, se encontró que en promedio en los SAF tradicionales el porcentaje de especies de plantas útiles puede variar del 75\% para el SAF milpachichipera (Moreno-Calles et al., 2012), hasta un 96\% que es el caso del SAF kuojtaliloyan (Toledo y Moguel, 2012). Asimismo, para los huertos, Caballero et al. (2010) reportan 1,400 especies de plantas útiles, las cuales constituyen el $25 \%$ de todas las plantas útiles documentadas en la Base de Datos de Plantas Útiles de México (Caballero y Cortés, 2012). Los recursos que alojan los SAF estudiados se destinan a satisfacer 15 diferentes tipos de usos (Apéndice 1), destacan alimenticios (principalmente frutos), forrajero y medicinal. En menor medida pero también relevantes: ornamental, materiales de construcción, combustibles, elaboración de bebidas alcohólicas, forrajes, utensilios, fibras, resinas útiles y látex, colorantes, venenos, producción de saponinas, rituales y para intercambio o venta. En algunos de los sistemas analizados se encontraron ejemplos de formas muy complejas de cultivo, así como formas de manejo incipiente, la mayor parte de las especies que se registraron en los SAF analizados son toleradas (Apéndice 1); es decir, son elementos de la vegetación natural circundante que se encontraban en los sitios abiertos para establecer el SAF, o que se reclutaron durante el proceso de manejo y la gente decidió mantenerlas ahí por alguna razón (Alcorn, 1990; Casas et al., 2007; Blancas et al., 2010). Le siguen en número las especies protegidas, las cuales además de dejarse en pie reciben algún tipo de cuidado, como por ejemplo la protección contra herbívoros, desyerbes, riego o aplicación de fertilizantes, entre otros. Finalmente, se identificaron especies que son propagadas vegetativamente o por medio de semillas, con el fin deliberado de aumentar su densidad poblacional. Estos son patrones comunes en diversos sistemas, registrados en estudios de los SAF tradicionales (Moreno-Calles et al., 2012), pero que requieren mayor profundización. No obstante, con la información disponible, aún se desconoce el número preciso de las especies que tienen algún tipo de manejo, así como los detalles de tales formas de manejo en los sistemas analizados. Esta es una línea de investigación prioritaria, que permitiría recuperar la experiencia mesoamericana milenaria para atender necesidades técnicas actuales en manejo de los recursos vegetales (Blancas et al., 2010; Moreno-Calles et al., 2012). Los estudios de caso y las revisiones sistemáticas de la información acerca de la producción y manejo a nivel de especies y de prácticas agroforestales permitirán evaluar con mayor detalle la importancia de los SAF en la satisfacción de las necesidades de subsistencia de las familias y comunidades rurales, así como su aporte potencial a la satisfacción de requerimientos a otras escalas.

Beneficios ecosistémicos de los SAF. Autores como Shibu (2009) han identificado un importante papel de la diversidad biocultural albergada en los SAF tradicionales en diversos beneficios ecosistémicos relevantes para la humanidad. Por ejemplo, de acuerdo a esta revisión, se identificó que de las 112 especies principales documentadas, el $84 \%$ de ellas son nativas a México (Apéndice 1). Además se registraron 15 tipos de beneficios de los diferentes SAF analizados: (1) retención del suelo, (2) delimitación de terrenos, (3) sombra, (4) barreras rompe-vientos y amortiguamiento del efecto de huracanes, (5) incremento de la fertilidad del suelo, (6) recuperación de la vegetación, (7) hábitat facilitador de otras especies, (8) control de plagas, (9) especies atrayentes o bioinsecticidas, (10) mantenimiento de fuentes de agua, (11) mejoramiento del microclima, (12) control de incendios, (13) ambiente propicio como reservorio de polinizadores, (14) abasto de diversos recursos, (15) indicador climático (Apéndice 1). Asimismo, otros valores culturales reconocidos explícitamente por la gente local en algunos estudios de caso es su capacidad "para atraer la lluvia o llamar el agua", y como "lujo", un término que define el embellecimiento del paisaje por parte de las plantas y los grupos de plantas. Otro beneficio evidente en todos los SAF examinados es la minimización del riesgo en el abasto de recursos, por medio de la estrategia múltiple de uso y el manejo de numerosas especies con los mismos usos y funciones (Alcorn, 1990; Toledo, 1990). El mantenimiento de un alta reserva de diversidad genética intraespecífica es un beneficio adicional que se ha documentado en el manejo de varias especies de cactáceas columnares en los sistemas agroforestales de milpa asociados a los bosques de cactáceas columnares en las zonas áridas del Valle de Tehuacán (Moreno-Calles et al., 2010), de los nopales en los huamiles en Guanajuato (Colunga García-Marín, 1984), o bien de los capulines en los metepantli de las zonas templadas de Tlaxcala y de Agave angustifolia Haw. en Jalisco (Vargas-Ponce et al., 2009). También estos sistemas agroforestales tradicionales son reservorios de agrobiodiversidad nativa; como por ejemplo de las variedades de maíz nativo (Boege, 2008), o también de otras especies domesticadas como el frijol kokox, documentadas en los ekuaros de la región purhépecha (Mapes et al., 1990).

Generación de heterogeneidad a escala de hábitat y de paisaje. Los diversos mosaicos creados por los sistemas agroforestales presentados favorecen paisajes complejos, y la heterogeneidad de ambientes puede incrementar el número de especies e incluso favorecer el establecimiento 
de especies que no se encuentran en los sistemas naturales (Tscharntke et al., 2005). Además, frecuentemente estos sitios constituyen pequeñas unidades de manejo en los que la composición de especies de árboles y arbustos puede ser muy diferente entre ellas de acuerdo al manejo de cada agricultor, situación que favorece una mayor contribución de especies a nivel de paisaje (Bhagwat et al., 2008). Mientras que los agricultores en cada parcela bajo manejo agroforestal pueden tener no más de 40 especies silvestres y cultivadas, a nivel de paisaje lo que encontramos para cada sistema es el manejo de un importante número de especies que puede ir desde 40 (sistemas intensivos chinampa y calal) hasta 400 especies (te lom, kuojtakiloyan y huertos). Además, son incorporadas en estos sistemas especies introducidas de alto valor para las comunidades locales; o bien, especies nativas a nivel regional que no estaban presentes en los sistemas de bosques originales (Moreno-Calles et al., 2010, 2012).

Disminución de la deforestación y de la presión sobre los remanentes de vegetación natural. Los sistemas agroforestales pueden ayudar a reducir la presión de la deforestación de nuevas áreas para la agricultura si se adoptan como alternativas a otras prácticas de uso de la tierra menos sustentables, como es la agricultura altamente intensificada, las plantaciones y monocultivos. También pueden ser fuentes de extracción de recursos forestales (Schroth et al., 2004), y éstos pueden aún incrementarse en las zonas agroforestales mediante prácticas de manejo y domesticación con base en el rico acervo de conocimientos tradicionales acerca del uso y manejo de las especies que pudimos revisar a lo largo del texto (Simons y Leakey, 2004).

La restauración de la biodiversidad. Los campesinos, frecuentemente, han desarrollado estrategias locales para aminorar los efectos de las limitaciones de humedad, nutrientes, competencia o facilitación entre especies y el efecto de las plagas. Estas limitaciones suelen ser los mismos obstáculos que enfrenta la restauración ecológica (Vieira et al., 2009). "La diversidad genera diversidad", es una de las premisas para la restauración de la diversidad biológica mediante la reforestación con especies nativas (Shibu, 2009). Los sistemas agroforestales tradicionales que revisamos en este ensayo, manejan una elevada biodiversidad nativa y útil, y ello suele ser la base para el mantenimiento e incorporación de la biodiversidad denominada "asociada" (Vandermeer et al., 2002; Perfecto y Vandermeer, 2008). En numerosos sistemas agroforestales de barbecho, como es el caso del tlacolol en Guerrero y la kool en la Península de Yucatán, comúnmente se propagan o se toleran especies principalmente nativas y se generan prácticas agroforestales como los árboles aislados y el t'olche' para acelerar la recuperación de la fertilidad del suelo, disminuir el efecto de la erosión, de la insolación y del "splash" (golpeteo de las gotas de lluvia sobre el suelo). En el sistema de milpa-chichipera es posible encontrar especies de cactáceas columnares de alto valor cultural, su tolerancia en estos espacios permite el establecimiento de otras especies de menor valor cultural; cuando estos SAF son dejados en descanso, se favorece el proceso de recuperación de la vegetación y de la fertilidad del suelo (Moreno-Calles y Casas, 2010). Cuando las especies empleadas en estos SAF son de valor cultural y/o económico para las comunidades humanas que las manejan, se incrementa el éxito de la restauración (Rocheleau et al., 1988).

Riesgos y Estrategias para recuperar, mantener y defender los SAF tradicionales. Los SAF tradicionales se están transformando en sentidos no deseables, y los principales indicadores de ello son: (1) la disminución de las especies y prácticas agroforestales presentes en ellos; (2) la pérdida del conocimiento y/o abandono de las prácticas tradicionales, un proceso relacionado con la migración de los hombres y mujeres jóvenes, con el envejecimiento o muerte de la población campesina; (3) la intensificación agrícola que determina una disminución o pérdida de los períodos de descanso, de la cobertura vegetal, de los cultivos tradicionales y un incremento en el uso de maquinaría, agroquímicos y de la cobertura arable; y (4) la renta o venta de la tierra a particulares para el desarrollo de cultivos intensivos o para desarrollo de casas-habitación. No obstante de todos estos procesos, existen pueblos y organizaciones campesinas e instituciones académicas interesadas en mantener estos sistemas debido a su importancia biocultural, ecológica y económica descrita a lo largo del presente documento. En este sentido queremos aportar la reflexión al respecto de lo que consideramos pueden ser acciones que pueden sugerirse para favorecer estos esfuerzos. En primer término es importante desarrollar investigación sobre el estado actual de los sistemas agroforestales tradicionales, realizar precisiones acerca de su ubicación y extensión en el país, inventarios de su flora y fauna, de sus transformaciones y de la problemática particular que tiene cada uno de ellos actualmente. Estos sistemas pueden ser estudiados con intereses diversos a partir de las ciencias ambientales, sociales, biológicas, etc., y puedan funcionar además en la capacitación de profesionistas locales, o de organizaciones campesinas y no gubernamentales que impulsen proyectos en estos sitios. Además de la aportación de información específica sobre los SAF, la capacitación local y la formación de profesionistas comprometidos, es importante fortalecer las instituciones, expresadas como reglas de uso de los recursos o valoraciones sagradas alrededor de ello; a lo largo del texto hemos observado la importancia de las reglas comunitarias para el mantenimiento de los árboles en las parcelas, los cuales son considerados en numerosas comunidades y ejidos como recursos colectivos y se realizan acciones y reglas para su conservación, aprovechamiento, restauración y mejoramiento, es el caso de las reglas con relación al corte de sabinos en el 
estado de Tlaxcala o bien las restricciones encontradas en los SAF de bosques de cactáceas del Valle de Tehuacán. Finalmente, consideramos relevante poner en las discusiones académicas y políticas el tema del manejo de estos sistemas en particular y de los recursos forestales, para influir en el desarrollo de políticas más coherentes con el contexto y la riqueza biocultural nacional. A lo largo de este análisis hemos identificado que algunas políticas gubernamentales están incidiendo negativamente en la permanencia de este importante patrimonio nacional. Los sistemas agrícolas tradicionales, y desde luego, los sistemas agroforestales, tienen un importante lugar para la continuidad biocultural y para la defensa de los pueblos de México e incluso para la identidad nacional y mesoamericana.

\section{Agradecimientos}

Los autores agradecen a los pueblos de México por su generosidad para compartir la información revisada en este texto. Agradecemos a los revisores que tan amablemente nos hicieron sugerencias muy relevantes para la mejora de este texto. Nuestro agradecimiento a José Blancas, Narciso Barrera Bassols, Diana Lope Alzina, Janis Alcorn, Micheline Cariño, Marco Antonio Vásquez-Dávila, Pablo AlarcónChaires y Teresa Rojas Rabiela por brindarnos sugerencias bibliográficas relevantes. La primera autora agradece el apoyo económico de la Red de Etnoecología y Patrimonio Biocultural del proyecto del Consejo Nacional de Ciencia y Tecnología (CONACYT) número 166563 para desarrollar una estancia posdoctoral en el Centro de Investigaciones en Ecosistemas (CIEco-UNAM). Es una investigación realizada también gracias al programa UNAM-DGAPA-PAPIIT IA2032132 "Caracterización de sistemas agroforestales tradicionales de México desde un enfoque biocultural" PAPIIT IN205111-3 y CONACYT, Proyecto CB-2008-01-103551.

\section{Literatura Consultada}

Aguilar-Støen M., Moe S.R. y Camargo-Ricalde S.L. 2009. Home gardens sustain crop diversity and improve farm resilience in Candelaria Loxicha, Oaxaca, Mexico. Human Ecology 37:55-77.

Albores Z.B. 1995. Tules y Sirenas: El impacto Ecológico y Cultural de la Industrialización en el Alto Lerma. Colegio Mexiquense A.C., Gobierno del Estado de México-Secretaria de Ecología, México, D.F.

Alarcón-Chaires P. 2001. Ecología y Transformación Campesina en la Meseta P'urhépecha: Una Tipología Socio-ecológica de Productores Rurales de Nahuatzen, Michoacán. Universidad Michoacana de San Nicolás de Hidalgo, Secretaría de Difusión Cultural y Extensión Universitaria, Morelia.

Alcorn J.B. 1983. El Te 'lom huasteco: Presente, pasado y futuro de un sistema de silvicultura indígena. Biotica 8:315-331.

Alcorn J.B. 1984. Huastec Mayan Ethnobotany. University of Texas Press, Austin.

Alcorn J.B. 1990. Indigenous agroforestry systems in the Latin
American tropics. En: Altieri M. A. y Hecht S.B. Eds. Agroecology and Small Farm Development, pp. 203-218, CRC Press, Boca Raton.

Altieri M.A. y Toledo V.M. 2005. Natural resources management among small-scale farmers in semiarid lands: Building on traditional knowledge and agroecology. Annals of Arid Zone 44:365-385.

Altieri M.A. y Trujillo J. 1987. The agroecology of corn production in Tlaxcala, Mexico. Human Ecology 15:189-220.

Arias L. 1995. La producción milpera actual en Yaxcabá, Yucatán. En: Hernández E., Bello E. y Levy S. La Milpa en Yucatán: Un Sistema de Producción Agrícola Tradicional. Tomo 1, pp. 171-200, Colegio Postgraduados, Texcoco.

Armillas P. 1971. Gardens on swamps. Science 174:653-661.

Áviles M.V., Barrera A., Salazar G., Santiago M.L., Sosa E., Tenorio S. y Tovar M. 2012. Conocimiento tradicional y ritualidad en la montaña de Guerrero. Una aproximación desde las prácticas de policultivo y tejido de la palma. En: Argueta A. y Olivé L. Eds. Conocimiento Tradicional, Innovación y Reapropiación Social, pp. 95-120, Siglo XXI Editores, México, D.F.

Barrera-Cristóbal H. 1999. Tlacolol: Sistema agroforestal del trópico seco. Tesis licenciatura, Universidad Autónoma Chapingo, Texcoco, 55 pp.

Barrera-Bassols N. y Toledo V.M. 2005. Ethnoecology of the Yucatec Maya: Symbolism, knowledge and management of natural resources. Journal of Latin American Geography 4:9-41.

Benz B.F., Santana M.F., Pineda L.R., Cevallos E.J., Robles H.L. y de Niz L.D. 1994. Characterization of mestizo plant use in the Sierra de Manantlan, Jalisco-Colima, Mexico. Journal of Ethnobiology 14:23-41.

Bergmann J.F. 1969. The distribution of cacao cultivation in preColumbian America. Annals of the Association of American Geographers 59:85-96.

Bernice B. 2010. El Desarrollo Rural en México y la Serpiente Emplumada. Fondo de Cultura Económica, Centro de Investigación de Estudios Avanzados, Instituto Politécnico Nacional, México D.F.

Bhagwat S.A., Willis K.J., Birks H.J.B. y Whittaker R.J. 2008. Agroforestry: a refuge for tropical biodiversity? Trends in Ecology and Evolution 23:261-267.

Blancas J., Casas A., Lira R. y Caballero J. 2009. Traditional management and morphological patterns of Myrtillocactus schenckii (Cactaceae) in the Tehuacán Valley, Central Mexico. Economic Botany 63:375-387.

Blancas J., Casas A., Rangel-Landa S., Moreno-Calles A., Torres I., Pérez-Negrón E., Solís L., Delgado-Lemus A., Parra F., Arellanes Y., Caballero J., Cortés L., Lira R. y Dávila P. 2010. Plant management in the Tehuacán-Cuicatlán Valley, Mexico. Economic Botany 64:287-302.

Blancas J.J., Parra F., Lucio J.D., Ruíz-Durán M.E., Pérez-Negrón E., Otero-Arnaiz A., Pérez-Nasser N. y Casas A. 2006. Manejo tradicional y conservación de la biodiversidad de Polaskia spp. (Cactaceae) en México. Zonas Áridas 10:20-40.

Blanckaert I., Swennen R.L., Paredes Flores M., Rosas López R. y Lira Saade R. 2004. Floristic composition, plant uses and management practices in homegardens of San Rafael Coxcatlán, Valley of Tehuacán-Cuicatlán, Mexico. Journal of Arid Environments 57:179-202.

Boege E. 2008. El Patrimonio Biocultural de los Pueblos Indígenas de México: Hacia la Conservación in situ de la Biodiver- 
sidad y Agrodiversidad en los Territorios Indígenas. Instituto Nacional de Antropología e Historia, Comisión Nacional para el Desarrollo de los Pueblos Indígenas México, D.F.

Breceda A., Arriaga L. y Coria R. 1997. Características socioeconómicas y uso de recursos naturales en los oasis. En: Arriaga L. y Rodríguez-Estrella R. Eds. Los Oasis de la Península de Baja California, pp. 261-284, Centro de Investigaciones Biológicas del Noroeste, La Paz.

Caballero J. y Cortés L. 2012. Base de Datos Etnobotánicos de Plantas de México (BADEPLAM). Jardín Botánico Instituto de Biología- Universidad Nacional Autónoma de México, México, D.F.

Caballero J., Cortés L. y Martínez-Balleste A. 2010. El manejo de la biodiversidad en los huertos familiares. En: Toledo V.M. Coord. La Biodiversidad de México. Inventarios, Manejos, Usos, Informática, Conservación e Importancia Cultural, pp. 220-234, Fondo de Cultura Económica, Consejo Nacional para la Cultura y las Artes, México, D.F.

Cariño M., Castorena L., Maya Y., Wurl J., Urciaga J. y Breceda A. 2012. Transformación de los ecosistemas áridos para su uso agrícola en Baja California Sur. Historia Agraria 56:81-106.

Carmona A. y Casas A. 2005. Management, phenotypic patterns and domestication of Polaskia chichipe (Cactaceae) in the Tehuacán Valley, Central Mexico. Journal of Arid Environments 60: $115-132$.

Casas A., Caballero J. y Valiente-Banuet A. 1999. Use, management and domestication of columnar cacti in south-central Mexico: a historical perspective. Journal of Ethnobiology 19:71-95.

Casas A., Viveros J.L. y Caballero J. 1994. Etnobotánica Mixteca: Sociedad, Cultura y Recursos Naturales en la Montaña de Guerrero. Instituto Nacional Indigenista, México, D.F.

Casas A., Caballero J., Mapes C. y Zárate S. 1997. Manejo de la vegetación, domesticación de plantas y origen de la agricultura en Mesoamérica. Boletín de la Sociedad Botánica de México 61:31-47.

Casas A., Otero-Arnaiz A., Pérez-Negrón E. y Valiente-Banuet A. 2007. In situ management and domestication of plants in Mesoamerica. Annals of Botany 100:1101-1115.

Casas A, Cruse-Sanders J., Morales E., Otero-Arnaiz A. y Valiente-Banuet A. 2006. Maintenance of phenotypic and genotypic diversity in managed populations of Stenocereus stellatus (Cactaceae) by indigenous peoples in Central Mexico. Biodiversity and Conservation 15:879-898.

Casas A., Valiente-Banuet A., Solís L. y Pérez-Negrón E. 2010. El manejo de la biodiversidad en el desierto: el Valle de TehuacánCuicatlán. En: Toledo V.M. Coord. La Biodiversidad de México. Inventarios, Manejos, Usos, Informática, Conservación e Importancia Cultural, pp. 235-272, Fondo de Cultura Económica/, Consejo Nacional para la Cultura y las Artes, México, D.F.

Casas A., Rangel-Landa S., Torres-García I., Pérez-Negrón, E., Solís L., Parra F., Delgado A., Blancas J., Farfán-Heredia B. y Moreno-Calles. A.I. 2008. In situ management and conservation of plant resources in the Tehuacán-Cuicatlán Valley, Mexico: An ethnobotanical and ecological perspective. En: de Albuquerque U.P. y Alves-Ramos M. Eds. Current Topics in Ethnobotany, pp. 1-23, Research Sigpost, Kerala.

Cervantes S.J.M. y Rojas R. T. 2000. Introducción del lirio acuático (Eichhornia crassipes) a México durante el porfiriato. Quipu 13:177-190.
Coe S.D. y Coe M.D. 1999. La Verdadera Historia del Chocolate. Fondo de Cultura Económica, México, D.F.

Colunga García-Marín P. 1984. Variación morfológica, manejo agrícola y grados de domesticación de Opuntia spp. en el Bajío Guanajuatense. Tesis maestría, Colegio de Postgraduados, Texcoco, 204 pp.

CDI. Comisión para el Desarrollo de los Pueblos Indígenas. 2012. Atlas de los Pueblos Indígenas de México <www.cdi.gob.mx/ index.php?option $=$ com_wrapper\&view $=$ wrapper $\&$ Itemid $=200$ 027> (consultado 6 de abril 2013).

Crews T.E. y Gliessman S.R. 1991. Raised field agriculture in Tlaxcala, Mexico: An ecosystem perspective on maintenance of soil fertility. American Journal of Alternative Agriculture 6:9-16.

Dahlin B. 1985. La geografía histórica de la antigua agricultura maya. En: Rojas R.T. y Sanders W.T. Eds. Historia de la Agricultura. Época Prehispánica-Siglo XVI, pp. 125-196, Instituto Nacional de Antropología e Historia, Secretaría de Educación Pública, México, D.F.

Dávila P., Arizmendi M.C., Valiente-Banuet A., Villaseñor J.L., Casas A. y Lira R. 2002. Biological diversity in the Tehuacán-Cuicatlán Valley, Mexico. Biodiversity and Conservation 11:421-442.

Denevan W.M. 1970. Aboriginal drained-field cultivation in the Americas. Science 169:647-654.

Denevan W.M. 1992. The pristine myth: The landscape of the Americas in 1492. Annals of the Association of American Geographers 82:369-385.

Donkin R.A. 1979. Agricultural Terracing in the Aboriginal New World. Wenner-Gren Foundation for Anthropological Research, University Arizona Press, Tucson.

Doolittle W.E. 2004. Garden are us, we are nature: Transcending antiquity and modernity. Geographical Review 94:391-404.

Emerson R.A. 1953. A preliminary survey of the milpa system of maize culture as practiced by the Maya Indians of the northern part of the Yucatan Peninsula. Annals of the Missouri Botanical Garden 40:51-62.

Evans S.T. 1990. The productivity of maguey terrace agriculture in central Mexico during the Aztec period. Latin American Antiquity 1:117-132.

Fernandes E.C.M. y Nair P.K.R. 1986. An evaluation of the structure and function of tropical homegardens. Agricultural Systems 21:279-310.

Flores, J. S., Tun Garrido, J., Ortiz Díaz, J.J. y Kantún Balam, J. 2010. Plantas Usadas en Cercas Vivas en la Península de Yucatán. Universidad Autónoma de Yucatán, Consejo Nacional de Ciencia y Tecnología, Mérida.

Flores-Guido J.S. 1998. Etnobotánica de las leguminosas en la península de Yucatán: Uso y manejo entre los mayas. Tesis Doctoral, Facultad de Ciencias, Universidad Nacional Autónoma de México, México, D.F., 168 pp.

Flores, J.S. y Espejel I. 1994. Tipos de Vegetación de la Península de Yucatán. Etnoflora Yucatanense. Universidad Autónoma de Yucatán, Mérida.

Frederick C.D. 2007. Chinampa cultivation in the basin of Mexico. Studies in Human Ecology and Adaptation 3:107-124.

García-Martínez L.E. 2012. Aspectos socioecológicos para el manejo sustentable del copal en el ejido de Acateyahualco, Gro. Tesis licenciatura, Centro de Investigaciones en Ecosistemas, Universidad Nacional Autónoma de México, Morelia, 97 pp. 
García-Cook A. 1974. Una secuencia cultural para Tlaxcala. Comunicaciones 10:5-22.

Gillespie A.R., Bocanegra-Ferguson D.M. y Jimenez-Osornio J.J. 2004. The propagation of Ramón (Brosimum alicastrum Sw.; Moraceae) in Mayan homegardens of the Yucatan Peninsula of Mexico. New Forests 27:25-38.

Gómez-Pompa A. 1987. On Maya silviculture. Estudios Mexicanos 1:1-17.

Gómez-Pompa A. 1991. Learning from traditional ecological knowledge: Insights from Mayan silviculture. En: GómezPompa A, Whitmore T.C. y Hadley M. Eds. Rain Forest Regeneration and Management, pp. 335-341, Organización de las Naciones Unidas para la Educación, The Parthenon Publishing Group, París.

Gómez-Pompa A., Flores J.S. y Fernández M.A. 1990. The sacred cacao groves of the Maya. Latin American Antiquity 1:247-257.

Gómez-Pompa A., Flores J.S. y Sosa V. 1987. The'pet kot': a manmade tropical forest of the Maya. Interciencia 12:10-15.

González-Jácome A. 1999. Los calales del suroeste de Tlaxcala: Una descripción del sistema en la Cuenca del río Zahuapan. En: Jiménez-Osorio J.J.. Ed. Los Camellones y Chinampas Tropicales, pp. 69-91, Universidad Autónoma de Yucatán, Mérida.

González-Jácome A. 2003. Cultura y Agricultura: Transformaciones en el Agro Mexicano. Universidad Iberoamericana, México, D.F.

González-Jácome A. 2008. Humedales en el Suroeste de Tlaxcala: Agua y Agricultura en el Siglo XX. Universidad Iberoamericana, México, D.F.

González -Jácome A. 2011. Historias Varias: Un Viaje en el Tiempo con los Agricultores Mexicanos. Universidad Iberoamericana, México, D.F.

Hernández F. [1790] 1942. Historia de Plantas de Nueva España. Instituto de Biología, Universidad Nacional Autónoma de México, México, D.F.

Hernández-Xolocotzi E. y Padilla R. 1980. Seminario sobre Producción Agrícola en Yucatán. Gobierno del estado de Yucatán, Secretaría de Programación y Presupuesto y Colegio de Postgraduados, Mérida.

Hernández-Xolocotzi E. 1977. Agroecosistemas de México: Contribuciones a la Enseñanza, Investigación y Divulgación Agrícola. Colegio de Postgraduados, Texcoco.

Hoogesteger-van Djik V.M. 2012. Tajos de la Sierra Gorda Guanajuatense: agroecosistemas de importancia ecológica, económica y cultural. Tesis licenciatura, Escuela Nacional de Estudios Superiores, Unidad Morelia, Universidad Nacional Autónoma de México, Morelia, 147 pp.

Jiménez-Osornio J. y Gómez-Pompa A. 1987. Las chinampas mexicanas. Pensamiento Iberoamericano 12:201-214.

Krishnamurthy L. y Ávila M. 1999. Agroforestería Básica. Programa de Naciones Unidas para el Medio Ambiente, Red de Formación Ambiental para América Latina y el Caribe. México, D.F.

Kumar B.M. y Nair P.K.R. 2004. The enigma of tropical homegardens. Agroforestry Systems 62:135-152.

Lambert D.P. 1996. Crop diversity and fallow management in a tropical deciduous forest shifting cultivation system. Human Ecology 24:427-453.

Linares O.F. 1976. "Garden hunting" in the American tropics. $\mathrm{Hu}$ man Ecology 4:331-349.
Lira R., Casas A., Rosas-López R., Paredes-Flores M., Pérez-Negrón E., Rangel-Landa S. Solís L., Torres I. y Dávila P. 2009. Traditional knowledge and useful plant richness in the Tehuacán-Cuicatlán Valley, México. Economic Botany 63:271-287.

Lot A., Novelo A. y Quiroz A. 1979. The chinampa: An agricultural system that utilizes aquatic plants. Journal of Aquatic Plant Management 17:74-75.

MacNeish R.S. 1967. Summary of the subsistence. En: Byers D.S. The Prehistory of the Tehuacan Valley: Environment and Subsistence, pp. 290-310, University of Texas Press, Austin.

Magaña A.M.A. 2006. Catálogo de Nombres Vulgares y Científcos de Plantas de Tabasco. $2^{\mathrm{a}}$ ed. Universidad Juárez Autónoma de Tabasco, Villahermosa.

Magdaleno M.L., García M.E., Valdés-Hernández J.I. y de la Cruz I.V. 2005. Evaluación del sistema agroforestal "árboles en terrenos de cultivo" en Vicente Guerrero, Tlaxcala, México. Revista Fitotecnia Mexicana 28:203-212.

Mapes C., Toledo V.M., Barrera-Bassols N. y Caballero J. 1990. La agricultura en una región indígena: la cuenca del lago de Pátzcuaro: En: Rojas R.T. Coord. Agricultura Indígena: Pasado y Presente., pp. 275-341, Centro de Investigaciones y Estudios en Antropología Social, Ediciones de la Casa Chata Núm. 27, México, D.F.

Mariaca-Méndez R., González-Jacome A. y Arias-Reyes L.M. 2010. El Huerto Maya Yucateco en el Siglo XVI. El Colegio de la Frontera Sur, Centro de Investigación y de Estudios Avanzados-Mérida, Universidad Intercultural Maya de Quintana Roo, Consejo Nacional de Ciencia y Tecnología-Yucatán, Fondos Mixtos de Fomento a la Investigación Científica y TecnológicaYucatán, México, D.F.

Martínez M. 1979. Catálogo de Nombres Vulgares y Científicos de Plantas Mexicanas. Fondo de Cultura Económica, México, D.F.

Maya Y., Coria R. y Domínguez R. 1997. Caracterización de los oasis. En: Arriaga L. y Rodríguez E.R. Eds. Los Oasis de la Península de Baja California, pp. 5-25, Centro de Investigaciones Biológicas del Noroeste, La Paz.

McNeil C.L. 2009. Chocolate in Mesoamerica: A Cultural History of Cacao. University Press of Florida, Gainesville.

Merlín-Uribe Y., González-Esquivel C.E., Contreras-Hernández A., Zambrano L., Moreno-Casasola P. y Astier M. 2013. Environmental and socio-economic sustainability of chinampas (raised beds) in Xochimilco, Mexico City. International Journal of Agricultural Sustainability 11:216-233.

Moguel P. y Toledo V.M. 1999. Biodiversity conservation in traditional coffee systems of Mexico. Conservation Biology 13:11-21.

Moguel P. y Toledo V.M. 2004. Conservar produciendo: Biodiversidad, café orgánicoy jardines productivos. Biodiversitas 55:1-7.

Montagnini F. 2006. Homegardens of Mesoamerica: Biodiversity, food security, and nutrient management. En: Kumar B.M. y Nair P.K.R. Eds. Tropical Homegardens: A Time Tested Example of Sustainable Agroforestry, pp. 61-84, Springer, Dordrecht.

Moreno-Calles A.I. 2010. Sistemas agroforestales y manejo de la biodiversidad: el caso de las zonas áridas del valle de Tehuacán. Tesis doctoral, Centro de Investigaciones en Ecosistemas, Universidad Nacional Autónoma de México, Morelia, Michoacán, $115 \mathrm{pp}$.

Moreno-Calles A.I. y Casas A. 2010. Agroforestry systems: Perspectives for restoration of semiarid zones of the Tehuacán Valley, Central Mexico. Ecological Restoration 28:361-368. 
Moreno-Calles A.I., Casas A., García-Frapolli E. y Torres-García I. 2012. Traditional agroforestry systems of multi-crop "milpa" and "chichipera" cactus forest in the arid Tehuacan Valley, Mexico: Their management and role in people's subsistence. Agroforestry Systems 84:207-226.

Moreno-Calles A.I., Casas A., Blancas J., Torres I., Masera O., Caballero J., García-Barrios L., Pérez-Negrón E. y Rangel-Landa S. 2010. Agroforestry systems and biodiversity conservation in arid zones: The case of the Tehuacán Valley, Central México. Agroforestry Systems 80:315-331.

Mountjoy D.C. y Gliessman S.R. 1988. Traditional management of a hillside agroecosystems in Tlaxcala, México: An ecologically based maintenance system. American Journal of Alternative Agriculture 3:3-10.

Nabhan G.P., García J., Routson R., Routson K. y Cariño-Olvera, M. 2010. Desert oases as genetic refugia of heritage crops: Persistence of forgotten fruits in the mission orchards of Baja California, Mexico. International Journal of Biodiversity and Conservation 2:56-69.

Nair P.K.R. 1997. Directions in tropical agroforestry research: past, present, and future. Agroforestry Systems 38:223-246.

Niñez V. 1987. Household gardens: theoretical and policy considerations. Agricultural Systems 23:167-186.

Ogata N. 2003. Domestication and distribution of chocolate tree (Theobroma cacao L.) in Mesoamerica. En: Gómez-Pompa A., Allen M.F., Fedick S.L. y Jiménez-Osornio J.J. Eds. The Lowland Maya Area: Three Millennia at the Human-Wildland Interface, pp. 415-438, The Haworth Press Inc., Binghampton.

Otero-Arnaiz A., Casas A., Bartolo C., Pérez-Negrón E. y Valiente-Banuet A. 2003. Evolution of Polaskia chichipe (Cactaceae) under domestication in the Tehuacán Valley, Central Mexico: reproductive biology. American Journal of Botany 90:593-602.

Palerm J.V. 1997. La persistencia y expansión de sistemas agrícolas tradicionales: El caso del huamil en el Bajío mexicano. Monografías del Jardín Botánico de Córdoba 5:121-133.

Parra F., Pérez-Nasser N., Lira R., Pérez-Salicrup D. y Casas A. 2008. Population genetics and process of domestication of Stenocereus pruinosus (Cactaceae) in the Tehuacán Valley, México. Journal of Arid Environments 72:1997-2010.

Perfecto I. y Vandermeer J. 2008. Biodiversity conservation in tropical agroecosystems: A new conservation paradigm. Annals New York Academic Science 1134:173-200.

Pretty J., Adams B., Berkes F., de Athayde S.F., Dudley N., Hunn E., Maffi L., Milton K., Rapport D., Robbins P., Sterling E., Stolton S., Tsing A.,Vintinnerk E. y Pilgrim S. 2009. The intersections of biological diversity and cultural diversity: Towards integration. Conservation and Society 7:100-112.

Puig H. 1994. Agroforestry in Mexico: Can the past be a guarantee for the future? Cellular and Molecular Life Sciences 50:621-625.

Quiroz-Flores A., Miranda-Arce M.G. y Lot-Helgueras A. 2008. Estudio comparativo de algunas variables fisicoquímicas del agua en canales secundarios de Xochimilco con y sin Eichhornia crassipes (Martius) Solms-Laubach. Polibotánica 25:127-133.

Remmers G.G.A. y De Koeijer H. 1992. The t'olche, a Maya system of communally managed forest belts: the causes and consequences of its disappearance. Agroforestry Systems 18:149-177.

Remmers G.G.A. y Ucan E.E. 1996. La roza-tumba-quema maya:
Un sistema agroecológico tradicional frente al cambio tecnológico. Etnoecológica 3:97-109.

Rico-Gray V., García-Franco J.G., Chemas A., Puch A. y Sima P. 1990. Species composition, similarity, and structure of mayan homegardens in Tixpeual and Tixcacaltuyub, Yucatan, México. Economic Botany 44:470-487.

Rocheleau D.E., Weber F.R. y Field-Juma A. 1988. Agroforestry in Dryland Africa. International Council of Research in Agroforestry, Nairobi.

Rojas Rabiela, T. 1991. La agricultura en la época prehispánica. En: Rojas-Rabiela T. Coord. La Agricultura en Tierras Mexicanas desde sus Orígenes hasta Nuestros Días, pp. 15-138, Comisión Nacional para la Cultura y las Artes, Grijalbo S.A. de C.V., México, D.F.

Rojas Rabiela T. 1993. La Agricultura Chinampera: Compilación Histórica. $2^{\text {a }}$ ed. Universidad Autónoma Chapingo, Texcoco.

Rojas Rabiela T. y Sanders W.T. 1985. Historia de la Agricultura Época Prehispánica Siglo XVI. Instituto Nacional de Antropología e Historia, México, D.F.

Rojas Rabiela T., Martínez J.L. y Murillo D. 2009. Cultura Hidráulica y Simbolismo Mesoamericano del Agua en el México Prehispánico. Centro de Investigaciones y Estudios Superiores en Antropología Social, Instituto Mexicano de Tecnología del Agua, México, D.F.

Rzedowski J. 1993. Diversity and origins of the phanerogamic flora of Mexico. En: Ramamoorthy T.P., Bye R.A., Lot, A. y Fa J.E. Eds. Biological Diversity of Mexico: Origins and Distribution, pp. 129- 144, Oxford University Press, Nueva York.

Salgado-Mora M.G., Ibarra-Núñez G., Macías-Sámano J.E. y López-Báez O. 2007. Diversidad arbórea en cacaotales del Soconusco. Chiapas, México. Interciencia 32:763-768.

Sanders W.T. 1993. El lago y el volcán. La chinampa. En: Rojas-Rabiela Comp. La Agricultura Chinampera: Compilación Histórica, pp. 129-179, Universidad Autónoma Chapingo, Texcoco.

Schroth G., da Fonseca G.A.B., Harvey C.A., Gascon C., Vasconcelos H.L. y Izac A.N. 2004. Agroforestry and Biodiversity Conservation in Tropical Landscapes. Island Press, Washington, DC.

Shibu J. 2009. Agroforestry for ecosystem services and environmental benefits: an overview. Agroforestry Systems 76:1-10.

Siemens A.H. y Puleston D.E. 1972. Ridged fields and associated features in Southern Campeche: New perspectives on the Lowland Maya. American Antiquity 37:228-239.

Simons A.J. y Leakey R.R.B. 2004. Tree domestication in tropical agroforestry. Agroforestry Systems 61:167-181.

Stephan-Otto E. 2005. Xochimilco Hoy: Una Realidad Insustentable. Universidad Nacional Autónoma de México, México, D.F.

Terán S. y Rasmussen C. 1994. La Milpa de los Mayas: La Agricultura de los Mayas Prehispánicos y Actuales del Noroeste de Yucatán. Gobierno del Estado de Yucatán, Mérida.

The International Plant Name Index (2012). http://www.ipni.org. (consultado noviembre 2012)

Thrupp L.A., Hecht S., Browder J.O., Lynch O.J., Megateli N. y O'Brien W. 1997. The Diversity and Dynamics of Shifting Cultivation: Myths, Realities, and Policy Implications. World Resources Institute, Washington, DC.

Toledo V.M. 1990. The ecological rationality of peasant production. En: Altieri M.A. y Hecth S.B. Eds. Agroecology and Small-farm Development, pp. 51-58, CRC Press, Boca Raton. 
Toledo V.M. 2002. Ethnoecology: a conceptual framework for the study of indigenous knowledge of nature. En: Stepp J.R., Wyndham F.S. y Zarger R.K. Ethnobiology and Biocultural Diversity: Proceedings of the 7th International Congress of Ethnobiology, pp. 511-522, University of Georgia, Athens.

Toledo V.M. y Barrera-Bassols N. 2008. La Memoria Biocultural. La Importancia Ecológica de las Sabidurías Tradicionales. Icaria Editorial, Barcelona.

Toledo V.M. y Moguel P. 2012. Coffee and sustainability: The multiple values of traditional shaded coffee. Journal of Sustainable Agriculture 36:353-377.

Toledo V.M., Barrera-Bassols N., García-Frapolli E. y AlarcónChaires P. 2008. Uso múltiple y biodiversidad entre los Mayas yucatecos. Interciencia 33:345-352.

Toledo V.M., Batis A.I., Becerra R., Martínez, E. y Ramos C.H. 1995. La selva útil: Etnobotánica cuantitativa de los grupos indígenas del trópico húmedo de México. Interciencia 20:177-187.

Toledo V.M., Ortiz-Espejel B., Cortés L., Moguel P. y Ordoñez M.D.J. 2003. The multiple use of tropical forests by indigenous peoples in Mexico: A case of adaptive management. Conservation Ecology 7:9.

Tscharntke T., Klein A.M., Kruess A., Steffan-Dewenter I. y Thies C. 2005. Landscape perspectives on agricultural intensification and biodiversity-ecosystem service management. Ecology Letters 8:857-874.

Vandermeer J., Lawrence D., Symstad A. y Hobbie S. 2002. Effect of biodiversity on ecosystem functioning in managed ecosystems. En: Loreau M., Naeem S. y Inchausti P. Eds. Biodiversity and Ecosystem Functioning, pp. 221-236, Oxford University Press, Oxford.

Vargas-Ponce O., Zizumbo-Villarreal D., Martínez-Castillo J., Coello-Coello J. y Colunga P. 2009. Diversity and structure of landraces of Agave grown for spirits under traditional agricultu- re: A comparison with wild populations of A. angustifolia (Agavaceae) and commercial plantations of A. tequilana. American Journal of Botany 96:448-457.

Vázquez-García J.A., Nieves H.G., Cházaro B.M., Vargas-Rodríguez Y.L., Flores M.A. y Luquín S.H. 2004. Listado preliminar de plantas vasculares del norte de Jalisco y zonas adyacentes. En: Vázquez-García J.A., Cházaro B.M.J., Nieves H.G., Vargas-Rodríguez Y.L., Vázquez G.M. y Flores M.A. Eds. Flora del Norte de Jalisco y Etnobotánica Huichola, pp. 116-168, Universidad de Guadalajara, Guadalajara.

Vieira D.L.M., Holl K.D. y Peneireiro F.M. 2009. Agro-successional restoration as a strategy to facilitate tropical forest recovery. Restoration Ecology 17:451-459.

West R.C. y Armillas P. 1950. Las chinampas de México. Poesía y realidad de los "jardines flotantes". Cuadernos Americanos 9:165-182.

Whitmore T.M. y Turner II B.L. 2001. Cultivated Landscapes of Middle America on the Eve of Conquest, Oxford University Press, Nueva York.

Wiersum K.F. 2004. Forest gardens as an "intermediate"land-use system in the nature-culture continuum: characteristics and future potential. Agroforestry Systems 61:123-134.

Wilken G.C. 1969. Drained-field agriculture: An intensive farming system in Tlaxcala, Mexico. Geographical Review 59:215241.

Wilken G.C.1987. Good Farmers: Traditional Agriculture Resources Management in México and Central America. University of California Press, Berkeley.

Williams L.S. 1990. Agricultural terrace evolution in Latin America. Yearbook. Conference of Latin Americanist Geographers 16:82-93.

Young A.M. 1994. The Chocolate Tree: A Natural History of Cacao. Smithsonian Institution Press, Washington, DC.

Recibido: 14 de noviembre de 2012

Aceptado: 12 de abril de 2013 